\title{
SOME REMARKS ON THE INTRINSIC MEASURES OF EISENMAN
}

\author{
BY \\ IAN GRAHAM $^{1}$ AND H. WU ${ }^{2}$
}

\begin{abstract}
This paper studies the intrinsic measures on complex manifolds first introduced by Eisenman in analogy with the intrinsic distances of Kobayashi. Some standard conjectures, together with several new ones, are considered and partial or complete answers are provided. Most of the counterexamples come from a closer examination of unbounded domains in complex euclidean space. In particular, a large class of unbounded hyperbolic domains are exhibited. Those unbounded domains of finite euclidean volume are also singled out for discussion.
\end{abstract}

1. Introduction. In his Berkeley thesis of 1969, D. A. Eisenman introduced on each complex manifold $M$ of dimension $n$ a $k$-dimensional measure $(1 \leqslant k \leqslant n)$ which depends only on the complex structure of $M$ (see [E]). The construction of these measures was modelled on the construction of the Kobayashi metric [K4] which had been available three years earlier (Kobayashi has also given an exposition of these measures in [K5] and has surveyed the results obtained about these measures up to 1975 in [K6]). These Eisenman measures are potentially very useful because they give a general frame of reference for the understanding of certain aspects of function theory on complex manifolds in general and on domains in $\mathbf{C}^{n}$ in particular. Moreover, when $k=n$ (top dimension), this top dimensional measure is the simplest known invariant of the complex structure of $M$. It is much to be regretted, therefore, that while a substantial amount of information has been amassed concerning the Kobayashi metric through the years, the present knowledge of the Eisenman measures has remained extremely meagre. Our puzzlement over this anomaly prompted us to take a closer look at these measures-particularly the top dimensional one-by examining systematically some of the open problems that have naturally emerged. This paper contains the results of this investigation.

Throughout the paper, there is a special emphasis on unbounded domains in $\mathbf{C}^{n}$, for it is our conviction that one must come to grips with these domains if one hopes to understand the notion of measure hyperbolicity. Although there are positive results in this paper, our explicit computations of the top dimension measures of these domains more often than not led to counterexamples of some existing conjectures. The somewhat unexpected phenomena uncovered in this paper may well be its main contribution.

\footnotetext{
Received by the editors May 18, 1984.

1980 Mathematics Subject Classification. Primary 32H99, 32H15; Secondary 53C55.

${ }^{1}$ Research done under partial support of the Natural Sciences and Engineering Research Council of Canada.

${ }^{2}$ Research done under partial support of the National Science Foundation.
} 
Let it be noted that the Eisenman construction, when suitably modified, also yields measures of each real dimension $k, 1 \leqslant k \leqslant 2 n$, on the $n$-dimensional complex manifold $M[\mathbf{E}]$. They are direct generalizations of the Kobayashi metric. Moreover, Eisenman also defined a sequence of "dual" measures (i.e., the Carathéodory measures, so to speak) in [E]. We will not discuss either of these in this paper.

We now give a more detailed description of the problems we have considered as well as an overview of this paper. First we fix our convention and terminology. The Eisenman $k$-norms $E_{k}$ are defined using the unit $k$-ball $B_{k}$ in $\mathbf{C}^{k}$; this is Eisenman's original convention but subsequent authors have sometimes employed the unit $k$-disc instead. The $k$-dimensional measure derived from $E_{k}$ is denoted by $I_{k}$. An $n$-dimensional complex manifold $M$ is $k$-measure hyperbolic iff $I_{k}(A)>0$ for any nonempty $k$-dimensional complex submanifold $A$ of $M ; M$ is strongly $k$-measure hyperbolic iff $E_{k}$, when applied to unit $k$-vectors on each compact subset of $M$, is bounded below by a positive constant. When $k=n$, the reference to $k$ will be dropped and we say simple measure hyperbolic and strongly measure hyperbolic. Again these definitions are by no means universal. Following a brisk review of such basic matters in $\S 2$, we make the observation in $\S 3$ that the product formula holds for the top dimensional norm: $E_{n+q}^{M \times P}=E_{n}^{M} \cdot E_{q}^{P}(\operatorname{dim} P=q)$. Since this equality is unlikely to hold if polydisks are used in place of balls in the definition of the $E_{k}$ 's, it makes for a very persuasive argument for adopting the definition using balls (in this sense, we have answered Problem B.2 on p. 401 of [K6]).

It is well known that if the holomorphic sectional curvature of a Hermitian manifold is bounded above by a negative constant, then $M$ is strongly 1-measure hyperbolic (theorem of Grauert-Reckziegel [G2]; cf. also [K5 and W1]); note that "strongly 1-measure hyperbolic" is equivalent to "hyperbolic" in the sense of Kobayashi [R2]. Moreover, a result of Kobayashi [K5, p. 121] says that $M$ is strongly measure hyperbolic if the Ricci curvature of $M$ is bounded above by a negative constant. It has been an obvious open problem (cf. e.g. [K6, p. 374]) whether there is a curvature condition to insure strong $k$-measure hyperbolicity that interpolates between the two preceding theorems. It turns out that a condition called strongly negative kth Ricci curature (Definition 4.4) does exactly this, so in a formal sense the preceding problem has been solved. In a deeper sense, however, the problem is still very much open because we do not know whether $M$ admitting a metric of strongly negative $k$ th Ricci curvature implies $M$ also admits a metric of strongly negative $(k+1)$ th Ricci curvature, while it is known that "strongly $k$-measure hyperbolic" implies "strongly $(k+1)$-measure hyperbolic" (cf. e.g. Proposition 2.17 below). Thus much remains to be done in this regard.

In $\$ 5$ we take up the well-known extension problem for measure hyperbolic manifolds [K7], namely, the following assertion:

(A) Let $X$ and $M$ be $n$-dimensional complex manifolds, let $V$ be a proper subvariety in $X$ and let $M$ be compact and measure hyperbolic. If $f: X-V \rightarrow M$ is holomorphic, then $f$ extends to a meromorphic map on $X$. 
Experience with extension theorems for hyperbolic manifolds (cf. [K1, K2 and K9]) leads one to believe that if (A) is indeed true, then so is the following:

$\left(\mathrm{A}^{\prime}\right)$ Let $X$ and $M$ be $n$-dimensional Hermitian manifolds, let $V$ be a proper subvariety of $X$ and let $M$ be compact. If $f: X-V \rightarrow M$ is holomorphic and $f$ is volume decreasing relative to the volume forms $\Omega_{X}$ and $\Omega_{M}$ of the corresponding Hermitian metrics, i.e., $f^{*} \Omega_{M} \leqslant \Omega_{X}$, then $f$ extends to a meromorphic map on $X$.

We could prove (A) only after making various additional hypotheses (Theorem $5.8)$. But the main thrust of $\S 5$ is perhaps the counterexample which shows that $\left(\mathrm{A}^{\prime}\right)$ is false even for Kählerian $X$ and $M$, and that one of the additional hypotheses in Theorem 5.8 is in some sense optimal (Example 5.15). This throws some doubt on the validity of (A) for a nonalgebraic manifold $M$. Very likely, the validity of $(\mathrm{A})$ for an algebraic $M$ would require the proof of another conjecture that an algebraic manifold is of general type iff it is measure hyperbolic [K7]. We note that this conjecture has recently been verified for algebraic surfaces by $M$. Green and Griffiths [G3] and D. Mumford and F. Bogomolov (for the latter we thank Robert Friedman for a private communication).

Next we take up the question of how regular the Eisenman norms $E_{k}$ have to be. While they are a priori only upper-semicontinuous, there was hope that they cannot behave too pathologically because they arise from holomorphic considerations. Specifically, on an $n$-dimensional complex manifold, one would like to have the following:

(B1) If $E_{n}$ vanishes on a nonempty open set, then it vanishes identically.

(B2) $\log E_{n}$ is plurisubharmonic.

These are respectively Problems B.6 and B.1 of [K6, pp. 401-402]. We show in $\S 6$ that both are false for domains in $\mathbf{C}^{n}$ and hence for noncompact manifolds. The case of a compact manifold is still open.

The question of the behaviour of hyperbolic manifolds under deformation is as old as the subject itself and has only recently been settled [B3 and B4]. In §7, we consider the corresponding question for measure hyperbolic manifolds. At least for domains in $\mathbf{C}^{n}$, every conceivable question in this context turns out to be false. Again, the compact case is unknown.

Among unbounded domains in $\mathbf{C}^{n}$, those that are closest to the bounded ones from the point of view of measure theory are obviously those with finite (euclidean) volume. Furthermore, there is reason to believe that measure hyperbolicity is intimately related to the finiteness of euclidean volume (cf. Proposition 8.1). It is then natural to consider the following series of assertions, which are arranged in such a way that each one implies the next:

(C1) There is a positive constant $\varepsilon$ such that if the unit ball in $\mathbf{C}^{n}$ is denoted by $B_{n}$ and $f: B_{n} \rightarrow \mathbf{C}^{n}$ is a holomorphic map whose Jacobian determinant $J f$ satisfies $J f(0)=1$, then the volume of $f\left(B_{n}\right)$ is bounded below by $\varepsilon$.

(C2) (Schottky-Landau theorem for domains of finite volume.) If $\Omega$ is a domain in $C^{n}$ of finite volume, then there exists a constant $R$ depending only on $\Omega$ such that if $B_{n}(r)$ is the ball of radius $r$ in $\mathbf{C}^{n}$ and $f: B_{n}(r) \rightarrow \Omega$ is a holomorphic map satisfying $J f(0)=1$, then $r \leqslant R$. 
(C3) If $\Omega$ is a domain of finite volume, then $\Omega$ is strongly measure hyperbolic.

(C4) If $\Omega$ is a domain of finite volume, then $\Omega$ is measure hyperbolic.

(C5) If $f: \mathbf{C}^{n} \rightarrow \mathbf{C}^{n}$ is a holomorphic map whose Jacobian determinant is not identically zero, then the volume of $f\left(\mathbf{C}^{n}\right)$ is infinite.

In $\S 8$, we give a counterexample to $(\mathrm{C} 3)$; hence $(\mathrm{C} 1)-(\mathrm{C} 3)$ are all false. The fact that $(\mathrm{C} 1)$ should fail took us by surprise. We are now inclined to believe that even (C5) may be false. See the discussion in $§ 8$.

The last section, $\$ 9$, of this paper is concerned with more examples of these measures on domains. We point out that as a result of the work of Eisenman $[\mathbf{P}]$, the intrinsic norm $E_{n}$ of the unit ball $B_{n}$, when restricted to the punctured unit ball $B_{n}-\{0\}$, differs from the intrinsic norm $E_{n}^{\prime}$ of the latter. This answers Problem B.4 of [K6, p. 402]. We also give a simple extension of Eisenman's work. In another vein, we exhibit some other domains with unusual intrinsic norm $E_{n}$ : a domain $\Omega$ which is not strongly measure hyperbolic but its $E_{n}$ is everywhere positive; a domain $\Omega_{1}$ which is hyperbolic outside a hypersurface $H$ and whose intrinsic norm $E_{n}$ vanishes exactly on $H$, and a domain $\Omega_{2}$ whose intrinsic norm $E_{n}$ vanishes on a set strictly bigger than a hypersurface. Finally, we show how to use a theorem of Sibony [S2] and a differential geometric calculation in [W4] to generate a large class of measure hyperbolic domains. A by-product of this is the observation that all (unbounded) strictly pseudoconvex domains are hyperbolic, a fact at once trivial and useful but never before mentioned anywhere else.

Above and beyond the results outlined above, our overall impression as a result of this work is that if the notion of measure hyperbolicity is to prove its usefulness, it should be along a different line from the one the development of hyperbolic manifolds has taken. There is no doubt that more examples of measure hyperbolic, but nonhyperbolic, manifolds are needed before we know what to expect of these measures. For this reason, we ask

(D) Can all unbounded measure hyperbolic domains in $\mathbf{C}^{2}$ be classified?

The need for new examples of measure hyperbolic manifolds is more acute in the compact case. If indeed the general belief that, for algebraic manifolds, measure hyperbolicity is equivalent to being of general type is correct (cf. the discussion in $\S 5$ ), then interesting compact measure hyperbolic manifolds would have to be nonalgebraic. The paucity of examples of nonalgebraic compact complex manifolds in general may then explain why it is so difficult to obtain new examples of compact measure hyperbolic manifolds.

We end this introduction by raising a question concerning what we call complete measure hyperbolicity. By definition, a measure hyperbolic domain $\Omega \subset \mathbf{C}^{n}$ is complete iff for each $p \in \partial \Omega$ and for each neighbourhood $U$ of $p$ in $\mathbf{C}^{n}, U \cap \Omega$ has infinite volume with respect to the intrinsic measure $I_{n}$ of $\Omega$. There are domains of holomorphy which are measure hyperbolic but not complete (the punctured disc in C, for instance). Nevertheless, the boundary estimates of the first author in [G1] show that every bounded strongly pseudoconvex domain is complete measure hyperbolic. The following is then a natural problem:

(E) Is a complete measure hyperbolic domain a domain of holomorphy? 
2. Basic definitions. In this section, we collect together some basic definitions as well as some elementary and mostly known facts needed for the succeeding sections.

Let $M$ be a complex manifold of dimension $n$. Let $p \in M$. We denote by $T_{p} M$ (resp. TM) the holomorphic tangent space to $M$ at $p$ (resp. the holomorphic tangent bundle). Let $\Lambda^{k} T M$ be the $k$ th exterior power of $T M$. The decomposable elements of $\Lambda^{k} T_{p} M$ (resp. $\left.\Lambda^{k} T M\right)$ will be denoted by $D_{p}^{k} M\left(\operatorname{resp} . D^{k} M\right)$. Thus $D_{p}^{k} M$ is the space of complex $k$-dimensional subspaces of $T_{p} M$ when nonzero scalar multiples are identified. If $\langle$,$\rangle is a Hermitian metric in T M$, it can be extended to a Hermitian metric on $\Lambda^{k} T M$ by defining: for $\alpha, \beta \in D_{p}^{k} M$ with $\alpha=v_{1} \wedge \cdots \wedge v_{k}$ and $\beta=w_{1}$ $\wedge \cdots \wedge w_{k}$, set

$$
\langle\alpha, \beta\rangle \equiv \operatorname{det}\left\{\left\langle v_{i}, w_{j}\right\rangle\right\}
$$

for $i, j=1, \ldots, k$, and extend this definition to arbitrary elements of $\Lambda^{k} T_{p} M$ by linearity. Write $\|\alpha\|^{2} \equiv\langle\alpha, \alpha\rangle$. Clearly if $\alpha$ in (2.1) contains a direction orthogonal to all the vectors in $\beta$ (we are identifying $\alpha$ with $\operatorname{span}_{\mathbf{C}}\left\{v_{1}, \ldots, v_{k}\right\}$ and $\beta$ with $\left.\operatorname{span}_{\mathbf{C}}\left\{w_{1}, \ldots, w_{k}\right\}\right)$, then $\langle\alpha, \beta\rangle=0$. For later use, we introduce

Definition 2.2. We say $\alpha, \beta \in D_{p}^{k} M$ are strictly orthogonal if any vector in $\alpha$ is orthogonal to any vector in $\beta$.

We denote the unit ball in $\mathrm{C}^{n}$ by $B_{n}$, but will always write $D$ in place of $B_{1}$ for the unit disc in $\mathbf{C}$. The standard inner product in $\mathbf{C}^{n}$ will always be understood unless otherwise stated. The complex manifold structure of $\mathbf{C}^{n}$ gives it a fixed orientation; by convention, we require the form

$$
\Theta_{n} \equiv(\sqrt{-1} / 2)^{n} d z_{1} \wedge d \bar{z}_{1} \wedge \cdots \wedge d z_{n} \wedge d \bar{z}_{n}
$$

to be positive. Modifying slightly a definition of Eisenman [E], we introduce

Definition 2.4. Let $k$ be any integer between 1 and $n$, and let $\alpha \in D_{p}^{k} M, p \in M$. The intrinsic (Eisenman) norm of $\alpha$ is by definition

$$
\begin{array}{r}
E_{k}(p ; \alpha) \equiv \inf \left\{\|\gamma\|^{2}: \gamma \in D_{0}^{k} B_{k}\right. \text { and there exists a holomorphic mapping } \\
\left.f: B_{k} \rightarrow M \text { such that } f(0)=p \text { and } f_{*}(\gamma)=\alpha\right\} .
\end{array}
$$

Equivalently, if we denote the ball of radius $R$ in $\mathbf{C}^{n}$ by $B_{n}(R)$, then

$$
\begin{aligned}
E_{k}(p ; \alpha) \equiv \inf \left\{R^{-2 k}: \text { there exists a holomorphic map } f: B_{k}(R) \rightarrow M\right. \\
\text { such that } \left.f(0)=p \text { and } f_{*}\left(\frac{\partial}{\partial z_{1}} \wedge \cdots \wedge \frac{\partial}{\partial z_{k}}(0)\right)=\alpha\right\} .
\end{aligned}
$$

In the above, if we not not require that the origin 0 be mapped onto $p$ by $f$, then we would need to take the norm $\|\gamma\|^{2}$ with respect to the Bergman metric of $B_{k}$ in (2.4.1), and to use a unit $k$-vector with respect to the Bergman metric in place of $\partial / \partial z_{1} \wedge \cdots \wedge \partial / \partial z_{k}(0)$ in (2.5). Observe that $E_{1}$ is just the square of the RoydenKobayashi infinitesimal metric [R2]. Also observe that there is nothing to be gained by using $B_{l}, l \geqslant k$, in place of $B_{k}$ in the right-hand side of (2.4.1). See Proposition 2.9(ii) on p. 58 of [E] for the simple proof. 
The function $E_{k}: D^{k} M \rightarrow[0, \infty)$ is upper-semicontinuous; Eisenman proved this for $k=n$ in [P, Lemma 2.5], and the proof for the general case of arbitrary $k$ is an easy consequence of the work of Royden ([R2 and R3]; see [S4] for a more general theorem). In general, $E_{k}$ is badly discontinuous; see $\S \S 6$ and 9 below for some nontrivial examples.

When $k=n$ (top dimension), yet another reformulation of (2.4.1) will be useful. Let $\left\{w_{1}, \ldots, w_{n}\right\}$ be complex coordinates near $p \in M$. Then

$$
\begin{aligned}
& E_{n}\left(p ; \frac{\partial}{\partial w_{1}}\right.\left.\wedge \cdots \wedge \frac{\partial}{\partial w_{n}}(p)\right) \\
&=\inf \left\{|J f(0)|^{-2}:\right. \text { there exists a holomorphic mapping } \\
&\left.\qquad f: B_{n} \rightarrow M \text { such that } f(0)=p\right\},
\end{aligned}
$$

where $J f$ denotes the complex Jacobian determinant of $f$. Now introduce the intrinsic volume form $\tau_{M}$ on $M$ defined by

$$
\tau_{M}(p)=E_{n}\left(p ; \frac{\partial}{\partial w_{1}} \wedge \cdots \wedge \frac{\partial}{\partial w_{n}}(p)\right)\left(\frac{\sqrt{-1}}{2}\right)^{n} d w_{1} \wedge d \bar{w}_{1} \wedge \cdots \wedge d w_{n} \wedge d \bar{w}_{n} .
$$

The following equivalent definition of $\tau_{M}$ shows that it is independent of the choice of coordinate systems: let $\Theta_{n}$ be as in (2.3); then

$$
\begin{aligned}
& \tau_{M}(p)=\inf \left\{\left(f^{*}\right)^{-1} \Theta_{n}(0):\right. \text { there is a holomorphic map } \\
& \left.f: B_{n} \rightarrow M \text { such that } f(0)=p \text { and } d f(0) \text { is nonsingular }\right\} .
\end{aligned}
$$

Again one may use the volume form of the Bergman metric of $B_{n}$ in place of $\Theta_{n}$ in (2.8), in which case the requirement that $f(0)=p$ on the right-hand side of (2.8) may be dropped.

More generally, let $A$ be a $k$-dimensional complex submanifold of an open set $U \subset M$ (call these local complex submanifolds of $M$ for short). We introduce an intrinsic volume form $\tau_{A}^{M}$ on $A$ as follows. Let $p \in A$ and let $w_{1}, \ldots, w_{n}$ be local coordinates near $p$ so that $\partial / \partial w_{1}, \ldots, \partial / \partial w_{k}$ are tangent to $A$ at $p$. Then by definition

$$
\tau_{A}^{M}(p) \equiv E_{k}\left(p ; \frac{\partial}{\partial w_{1}} \wedge \cdots \wedge \frac{\partial}{\partial w_{k}}(p)\right)\left(\frac{\sqrt{-1}}{2}\right)^{k} d w_{1} \wedge d \bar{w}_{1} \wedge \cdots \wedge d w_{k} \wedge d \bar{w}_{k} .
$$

Or equivalently,

$$
\begin{aligned}
& \tau_{A}^{M}(p)=\inf \left\{\left(f^{*}\right)^{-1} \Theta_{k}(0): f: B_{k} \rightarrow M\right. \text { is holomorphic, } \\
& \left.\qquad f(0)=p, d f(0) \text { is nonsingular, and } d f\left(T_{0} B_{k}\right)=T_{p} A\right\} .
\end{aligned}
$$

This shows the independence of the definition from the choice of $\left\{w_{i}\right\}$ in (2.9). It has already been remarked that $E_{k}$ is upper-semicontinuous. Hence $\tau_{A}^{M}$ is an integrable $2 k$ form on $A$. Now define the intrinsic volume of $A$ to be

$$
I_{k}(A) \equiv \int_{A} \tau_{A}^{M}
$$


In particular, the intrinsic volume of an open subset $U$ of $M$ is

$$
I_{n}(U) \equiv \int_{U} \tau_{M}
$$

The assignment $A \rightarrow I_{k}(A)$ defines a Borel measure on every $k$-dimensional complex submanifold of $M$; this is the Eisenman measure of dimension $k$. Following Eisenman [E], we introduce another Borel measure on each such $A$ as follows: let $\lambda_{k}$ be the Borel measure on $B_{k}$ defined by integration with respect to the volume element of the Bergman metric on $B_{k}$; then

$$
\begin{aligned}
I_{k}^{*}(A)=\inf \left\{\sum_{i=1}^{\infty} \lambda_{k}\left(E_{i}\right): \text { each } E_{i} \subset B_{k}\right. \text { and there exist } \\
\text { holomorphic maps } \left.f_{i}: B_{k} \rightarrow M \text { such that } A \subset \bigcup_{i=1}^{\infty} f_{i}\left(E_{i}\right)\right\} .
\end{aligned}
$$

Note that the measures $I_{k}$ and $I_{k}^{*}$ are both decreasing with respect to holomorphic mappings in the same way that the Kobayashi metric is. Moreover, on $B_{n}$, there are constants $c_{n, k}$ such that $I_{k}^{*}(A)=c_{n, k} I_{k}(A)$ for every local $k$-dimensional complex submanifold $A$ of $B_{k}$ (see [E, Proposition 1.5 on p. 51 and Proposition 2.4 on p. 57]). Standard reasoning then gives $I_{k}^{*} \leqslant c_{n, k} I_{k}$.

LEMMA 2.14. On any complex manifold of dimension $n$, the equality $I_{k}^{*}=c_{n, k} I_{k}$ holds.

For $n=k$ this has been proved in [E, Proposition 2.13]. The general proof is only a minor modification of Eisenman's argument provided one uses the upper-semicontinuity of $E_{k}$ alluded to above (this lemma was in fact conjectured in [E], but at that time the upper-semicontinuity of $E_{k}$ was not available).

Whereas most of the definitions in the study of hyperbolic manifolds have met with universal agreement, the notions of "measure hyperbolicity" and "strong measure hyperbolicity" are susceptible to tremendous variations (see [K5, K6 and P]). In order to standardize the terminology of this paper, we introduce

Definition 2.15. A complex manifold $M$ of dimension $n$ is said to be $k$-measure hyperbolic iff for any nonempty local $k$-dimensional complex submanifold $A$ of $M$, $I_{k}(A)>0 . M$ is strongly $k$-measure hyperbolic iff for any compact set $K \subset M$, there is a positive constant $c_{K}$ such that $E_{k}(p ; \alpha) \geqslant c_{K}\|\alpha\|^{2}$ for all $p \in K$ and for all $\alpha \in D_{p}^{k} M$.

In general, strong $k$-measure hyperbolicity is a more stringent requirement than $k$-measure hyperbolicity (see $\$ 9$ ). When $k=n$, which is the most important special case in this paper, we will simplify the terminology by using measure hyperbolicity and strong measure hyperbolicity in place of $n$-measure hyperbolicity and strong $n$-measure hyperbolicity. We will also say that $M$ is almost hyperbolic iff there exists a proper subvariety $V \subset M$ such that $M$ is hyperbolic at each point of $M-V$ in the sense that for each compact subset $K$ of $M-V$, there exists a positive constant $c_{K}$ 
such that $E_{1}(p ; X) \geqslant c_{K}\|X\|^{2}$ for all $p \in K, X \in T_{p} M$ (see [R2]). In general, we have the natural inclusions:

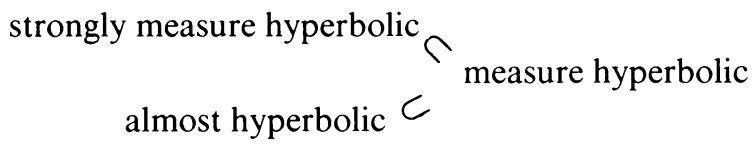

For the lower inclusion, see for instance Proposition 2.17 following. It is very tempting to conjecture that every strongly measure hyperbolic manifold is almost hyperbolic, but the evidence at hand in support of this conjecture is very limited. It is well known that all bounded domains are hyperbolic and hence strongly measure hyperbolic. On the other extreme, projective space $\mathbf{P}_{n} \mathbf{C}$ with certain hyperplanes deleted furnishes examples of unbounded hyperbolic domains and unbounded almost hyperbolic (but nonhyperbolic) domains in $\mathbf{C}^{n}$ (cf. [K3] for a survey). In this paper, we deal only with domains which are in between these two extremes: unbounded but with $C^{0}$ or $C^{\infty}$ boundary manifolds. In $\S 9$ will be found examples of domains with $C^{\infty}$ boundary which are (1) almost hyperbolic and the intrinsic volume form vanishes somewhere, and (2) not strongly measure hyperbolic but the intrinsic volume form is everywhere positive.

Purely for the sake of completeness, we state here the two characteristic properties of the intrinsic volume forms $\tau_{M}$ and the intrinsic norms $E_{k}^{M}$ (the superscript will serve to pin down the manifold under discussion).

LEMMA 2.16. (i) Let $\phi: M \rightarrow N$ be a holomorphic map between complex manifolds each of dimension $\geqslant k$. Then $E_{k}^{N}(\phi(p) ; d \phi(\alpha)) \leqslant E_{k}^{M}(p ; \alpha)$ for all $p \in M$ and for all $\alpha \in D_{p}^{k} M$.

(ii) If $M$ is the unit ball $B_{n}, n \geqslant k$, then $E_{k}^{M}(p ; \alpha)=\|\alpha\|^{2}$, where $\|\alpha\|$ denotes the norm of the Bergman metric on $B_{n}$.

The proof of (i) is trivial; the proof of (ii) can be found in [E, p. 57]. As usual $E_{k}^{M}$ can be characterized as the largest norm on $D^{k} M$ ( $M$ running through all complex manifolds) with these two properties (cf. [K5]). The next proposition gives a relationship between $E_{k}$ and $E_{k+1}$.

Proposition 2.17. Suppose $1 \leqslant k<n=\operatorname{dim} M$. If $M$ is strongly $k$-measure hyperbolic, then it is strongly $(k+1)$-measure hyperbolic. (The converse is obviously false.)

Proof. Introduce a Hermitian metric $\langle$,$\rangle in T M$, and hence also in $\Lambda^{\prime} T M$ for $l=2, \ldots, n$. Let $p \in M$ and let $\alpha \in D_{p}^{k+1} M$ such that $\|\alpha\|=1$. Suppose there exists a holomorphic map $f: B_{k+1} \rightarrow M$ such that $f(0)=p$ and $d f(\gamma)=\alpha$ for some $\gamma \in D_{0}^{k+1} B_{k+1}$ satisfying $\|\gamma\|=\varepsilon$. Since $\|\gamma\|=\varepsilon$ and $\|d f(\gamma)\|=1$, there must exist a tangent vector $u$ of $B_{k+1}$ at 0 such that $\|u\|=1$ while $\|d f(u)\| \leqslant \varepsilon^{-1 / k+1)}$. Write $\gamma=\gamma^{\prime} \wedge u$, where $\gamma^{\prime} \in D_{0}^{k} B_{k+1}$ and $\gamma^{\prime}$ is strictly orthogonal to $u$ (Definition 2.2). There is no loss of generality in regarding $\gamma^{\prime}$ as the tangent space of $B_{k}$ at 0 . We now use the following well-known algebraic fact (cf. [W3, p. 10]). 
Lemma 2.18. Let $1 \leqslant k, l \leqslant n$ and let $\alpha \in \Lambda^{k} T_{p} M, \beta \in \Lambda^{\prime} T_{p} M$. Then $\|\alpha \wedge \beta\| \leqslant$ $\|\alpha\| \cdot\|\beta\|$. If both $\alpha$ and $\beta$ are decomposable and $k+l \leqslant n$, then equality holds iff $\alpha$ is strictly orthogonal to $\beta$.

Returning to the proof of Proposition 2.17, we see that $\left\|\gamma^{\prime}\right\|=\varepsilon$ by Lemma 2.18. Again using this lemma, we obtain

$$
\begin{aligned}
1 & =\|d f(\gamma)\|=\left\|d f\left(\gamma^{\prime}\right) \wedge d f(u)\right\| \\
& \leqslant\left\|d f\left(\gamma^{\prime}\right)\right\| \cdot\|d f(u)\| \leqslant\left\|d f\left(\gamma^{\prime}\right)\right\| \cdot \varepsilon^{-1 \wedge k+1)} .
\end{aligned}
$$

Thus $\left\|\gamma^{\prime}\right\|=\varepsilon$ while $\left\|d f\left(\gamma^{\prime}\right)\right\| \geqslant \varepsilon^{1 / k+1)}$. If we set $\eta=\varepsilon^{-1 / k+1)} \gamma^{\prime}$, then $\eta \in D_{0}^{k} B_{k}$, $\|\eta\|=\varepsilon^{k \lambda(k+1)}$ and $\|d f(\eta)\|=1$.

Now if $M$ were not strongly $(k+1)$-measure hyperbolic, then we could contradict the fact that $M$ is strongly $k$-measure hyperbolic by taking a sequence of values $\varepsilon \rightarrow 0$ and using the above construction. Q.E.D.

The reader familiar with the Kobayashi metric will not be surprised by the following

LEMMA 2.19. If $M$ is a complex manifold and $\tilde{M}$ is a covering manifold with projection $\pi: \tilde{M} \rightarrow M$, then

$$
E_{k}^{\tilde{M}}(p ; \alpha)=E_{k}^{M}(\pi(p) ; d \pi(\alpha))
$$

for all $p \in \tilde{M}$ and for all $\alpha \in D_{p}^{k} \tilde{M}$.

We end this section with a few simple remarks on the standard examples. It has been pointed out above that on $B_{n}, E_{k}(p ; \alpha)=\|\alpha\|^{2}$ with $\|\alpha\|$ understood to be the Bergman norm [E], i.e., the norm of the Bergman metric $d s^{2}$ given by

$$
d s^{2}=\sum_{i, j=1}^{n}\left\{\frac{\left(1-\|z\|^{2}\right) \delta_{i j}+\bar{z}_{i} z_{j}}{\left(1-\|z\|^{2}\right)^{2}}\right\} d z_{i} d \bar{z}_{j}
$$

Its volume form and hence the intrinsic volume form $\tau_{n}\left(\equiv \tau_{B_{n}}\right)$ of $B_{n}$ is easily computed to be

$$
\tau_{n}=\frac{1}{\left(1-\|z\|^{2}\right)^{n+1}} \Theta_{n}
$$

(see (2.3)).

On the unit polydisc $\Delta_{n}$, we have much less information. $E_{1}$ is known because it is just the square of the Royden-Kobayashi infinitesimal metric and the latter is known to be just the Bergman metric on $\Delta_{n}$. Also, $E_{n}$ is known because it is equal to the volume form of the Bergman metric

$$
\tau_{\Delta_{n}}=\left(\frac{\sqrt{-1}}{2}\right)^{n} \prod_{i=1}^{n} \frac{d z_{i} \wedge d \bar{z}_{i}}{\left(1-\left|z_{i}\right|^{2}\right)^{2}}
$$


(this is a consequence of the automorphism invariance of $\tau_{\Delta_{n}}$ and the homogeneity of $\Delta_{n}$; see also Corollary 3.2 below). However, for $1<k<n$, nothing is known about $E_{k}^{\Delta_{n}}$ except for some special cases such as those covered by Theorem 3.1 below.

We now produce for each $n$ an $n$-dimensional compact complex manifold which is strongly measure hyperbolic but not $k$-measure hyperbolic for $1 \leqslant k<n$. Let $M$ be a compact complex manifold which is strongly measure hyperbolic, e.g., take $M$ to be the Fermat variety of degree $n+4$ in $\mathbf{P}_{n+1} \mathbf{C}, n \geqslant 1$ (cf. [K6, p. 394]). Let $\pi$ : $\tilde{M} \rightarrow M$ be the blow-up (quadratic transform) of $M$ at some point $p \in M$. Since $\mathrm{C}^{n-1} \subset \pi^{-1}(p) \subset \tilde{M}, \tilde{M}$ is not $k$-measure hyperbolic for any $k=1, \ldots, n-1$. On the other hand $\tilde{M}$ remains strongly measure hyperbolic because if $f: B_{n} \rightarrow \tilde{M}$ is a nondegenerate holomorphic map (i.e. $d f$ is somewhere nonsingular), so is $\pi \circ f$ : $B_{n} \rightarrow M$. Thus the assertion follows from (2.16) for the case $k=n$.

We can also use the Fermat varieties to show that the product of strongly $k$-measure hyperbolic manifolds need not in general be $k$-measure hyperbolic. In fact, let $F$ be the 2-dimensional Fermat surface of degree six (say) in $\mathbf{P}_{3} \mathbf{C}$. Then $F$ is strongly 2-measure hyperbolic. However $F \times F$ contains $\mathbf{P}_{1} \mathbf{C} \times \mathbf{P}_{1} \mathbf{C}$ (cf. [K6, p. 394]) and hence cannot be 2-measure hyperbolic.

It is straightforward to show that the product of (strongly) measure hyperbolic manifolds remains (strongly) measure hyperbolic. In the next section, we shall give a quantitative version of this statement.

3. The product formula. The main purpose of this section is to prove the following theorem.

THEOREM 3.1. Let $M$ and $N$ be complex manifolds of dimensions $m$ and $n$ respectively, and let $1 \leqslant k \leqslant m, 1 \leqslant l \leqslant n$. Let $p \in M$ and $q \in N$. Suppose $\alpha \in D_{(p, q)}^{k+l} M \times$ $N$ and $\alpha=\beta \wedge \gamma$, where $\beta \in D_{p}^{k} M$ and $\gamma \in D_{q}^{l} N$. Then

$$
E_{k+l}^{M \times N}((p, q) ; \alpha)=E_{k}^{M}(p ; \beta) \cdot E_{l}^{N}(q ; \gamma) .
$$

Corollary 3.2. Let $\tau_{M}, \tau_{N}$ and $\tau_{M \times N}$ denote the intrinsic volume forms of $M, N$ and $M \times N$ respectively. Then $\tau_{M \times N}=\tau_{M} \wedge \tau_{N}$.

In the preceding corollary as well as in the theorem, we have purposely omitted some projection maps in the interest of brevity. The corollary may be considered an answer to Problem B.2 on p. 401 of [K6]. The latter problem was formulated in terms of an intrinsic volume form defined by polydiscs; in that case this product formula is not likely to hold and in fact we do not believe there would be anything more than a pair of inequalities involving the two sides of (3.1.1).

ProOF OF THE THEOREM. We first prove $E_{k+l}^{M \times N}((p, q) ; \alpha) \leqslant E_{k}^{M}(p ; \beta) \cdot E_{l}^{N}(q ; \gamma)$; this proof is valid whether balls or polydiscs are used to define $E_{k}$. Given any $\varepsilon>0$, let $f_{1}: B_{k} \rightarrow M$ and $f_{2}: B_{l} \rightarrow N$ be holomorphic maps so that $f_{1}(0)=p, f_{2}(0)=q$, and for some $\omega_{1} \in D_{0}^{k} B_{k}$ and $\omega_{2} \in D_{0}^{l} B_{l}$, we have

$$
\begin{array}{ll}
d f_{1}\left(\omega_{1}\right)=\beta, & \left\|\omega_{1}\right\|^{2} \leqslant E_{k}^{M}(p ; \beta)+\frac{1}{2} \varepsilon, \\
d f_{2}\left(\omega_{2}\right)=\gamma, & \left\|\omega_{2}\right\|^{2} \leqslant E_{l}^{N}(q ; \gamma)+\frac{1}{2} \varepsilon .
\end{array}
$$


Now $B_{k+l} \subset B_{k} \times B_{l}$, so dispensing with the projection maps for the sake of notational simplicity, we have $\omega_{1} \wedge \omega_{2} \in D_{0}^{k+l} B_{k+l}$, and $f \equiv\left(f_{1}, f_{2}\right): B_{k+l} \rightarrow M \times$ $N$ is a holomorphic map such that $f(0)=(p, q)$ and $d f\left(\omega_{1} \wedge \omega_{2}\right)=\alpha$. Since $\left\|\omega_{1} \wedge \omega_{2}\right\|=\left\|\omega_{1}\right\| \cdot\left\|\omega_{2}\right\|$, we see that

$$
E_{k+l}^{M \times N}((p, q) ; \alpha) \leqslant\left\|\omega_{1}\right\|^{2} \cdot\left\|\omega_{2}\right\|^{2} \leqslant E_{k}^{M}(p ; \beta) \cdot E_{l}^{N}(q ; \gamma)+O(\varepsilon),
$$

where $O(\varepsilon) \rightarrow 0$ as $\varepsilon \rightarrow 0$. This proves the inequality.

Now we prove $E_{k}^{M}(p ; \beta) \cdot E_{l}^{N}(q ; \gamma) \leqslant E_{k+l}^{M \times N}((p, q) ; \alpha)$. This proof depends on the fact that the ball is round. Thus let $f: B_{k+l} \rightarrow M \times N$ be a holomorphic map such that $f(0)=(p, q)$, and for some $\omega \in D_{0}^{k+l} B_{k+l}$, we have

$$
d f(\omega)=\alpha, \quad \text { where }\|\omega\|^{2} \leqslant E_{k+l}^{M \times N}((p, q) ; \alpha)+\varepsilon .
$$

Let us write $\omega=a\left(\partial / \partial z_{1} \wedge \cdots \wedge \partial / \partial z_{k+l}\right)(0)$ for some $a \in \mathbf{C}, a \neq 0$. Note that $|a|=\|\omega\|$. After a unitary transformation of $\mathbf{C}^{k+l}$ (and hence of $\boldsymbol{B}_{k+l}$ onto itself) if necessary, we may assume that $d f\left(b \partial / \partial z_{1} \wedge \cdots \wedge \partial / \partial z_{k}(0)\right)=\beta$ for some $b \in \mathbf{C}$, $b \neq 0$. Since

$$
\begin{aligned}
\beta \wedge \gamma & =\alpha=d f(\omega) \\
& =d f\left(b \frac{\partial}{\partial z_{1}} \wedge \cdots \wedge \frac{\partial}{\partial z_{k}}(0)\right) \wedge d f\left(\frac{a}{b} \frac{\partial}{\partial z_{k+1}} \wedge \cdots \wedge \frac{\partial}{\partial z_{k+l}}(0)\right) \\
& =\beta \wedge d f\left(\frac{a}{b} \frac{\partial}{\partial z_{k+1}} \wedge \cdots \wedge \frac{\partial}{\partial z_{k+l}}(0)\right)
\end{aligned}
$$

it follows that

$$
d f\left(\frac{a}{b} \frac{\partial}{\partial z_{k+1}} \wedge \cdots \wedge \frac{\partial}{\partial z_{k+l}}(0)\right)=\gamma\left(\bmod \left\{\frac{\partial}{\partial z_{1}}(0), \ldots, \frac{\partial}{\partial z_{k}}(0)\right\}\right) .
$$

Now let $i_{1}: B_{k} \rightarrow B_{k+l}$ and $i_{2}: B_{l} \rightarrow B_{k+l}$ be the injections induced by the canonical injections $\mathbf{C}^{k} \equiv \mathbf{C}^{k} \times\{0\} \subset \mathbf{C}^{k+l}$ and $\mathbf{C}^{l} \equiv\{0\} \times \mathbf{C}^{l} \subset \mathbf{C}^{k+l}$. Also let $\pi_{1}: M \times N$ $\rightarrow M$ and $\pi_{2}: M \times N \rightarrow N$ be the canonical projections. If $f_{1} \equiv \pi_{1} \circ f \circ i_{1}$, and $f_{2} \equiv \pi_{2} \circ f \circ i_{2}$, then $f_{1}: B_{k} \rightarrow M$ and $f_{2}: B_{l} \rightarrow N$ are holomorphic maps such that $f_{1}(0)=p, f_{2}(0)=q$ and by (3.3),

$$
d f_{1}\left(b \frac{\partial}{\partial z_{1}} \wedge \cdots \wedge \frac{\partial}{\partial z_{k}}(0)\right)=\beta, \quad d f_{2}\left(\frac{a}{b} \frac{\partial}{\partial z_{1}} \wedge \cdots \wedge \frac{\partial}{\partial z_{l}}(0)\right)=\gamma .
$$

Hence,

$$
E_{k}^{M}(p ; \beta) \cdot E_{l}^{N}(q ; \gamma) \leqslant|b|^{2} \cdot|a / b|^{2}=|a|^{2}=\|\omega\|^{2} \leqslant E_{k+l}^{M \times N}((p, q) ; \alpha)+\varepsilon .
$$

Since $\varepsilon$ is arbitrary, we are done. Q.E.D.

Application. Let $D^{*}$ be the punctured disc, i.e. $D^{*}=\{0<|z|<1\}$. We shall compute the intrinsic volume form $\tau_{k, l}$ of $\Delta_{k, l} \equiv D^{*} \times \cdots \times D^{*} \times D \times \cdots \times D$ ( $k$ copies of $D^{*}$ and $l$ copies of $D$ ). We shall make use of Lemma 2.19.

We know that the intrinsic volume form of $D$ is $\left(1-|z|^{2}\right)^{-2}(\sqrt{-1} / 2) d z \wedge d \bar{z}$. Now the map $z \rightarrow \exp (-(1+z) /(1-z))$ gives a covering of $D$ onto $D^{*}$, and so by Lemma 2.19, the intrinsic volume form of $D^{*}$ is $\left(4(|z| \log |z|)^{2}\right)^{-1}(\sqrt{-1} / 2) d z \wedge d \bar{z}$. Thus by Corollary 3.2,

$$
\tau_{k, l}=\left(\frac{\sqrt{-1}}{2}\right)^{k+l} \prod_{i=1}^{k} \frac{d z_{i} \wedge d \bar{z}_{i}}{4\left(\left|z_{i}\right| \log \left|z_{i}\right|\right)^{2}} \wedge \prod_{i=k+1}^{k+l} \frac{d z_{i} \wedge d \bar{z}_{i}}{\left(1-\left|z_{i}\right|^{2}\right)^{2}}
$$


It follows that if for any $r$ such that $0<r<1$, we define $D(r)=\{|z|<r\}$ and $D^{*}(r)=D(r)-\{0\}$, then the region $D^{*}(r) \times \cdots \times D^{*}(r) \times D(r) \times \cdots \times D(r)$ ( $k$ and $l$ copies, as before) as a subset of $\Delta_{k, l}$ has finite volume with respect to $\tau_{k, l}$. This fact was first observed by Griffiths [G6] and is crucial in his work [G6 and G7] as well as in Kobayashi and Ochiai [K7].

4. Curvature condition for $k$-measure hyperbolicity. Let $M$ be an $n$-dimensional Hermitian manifold; the connection to be used will be understood to be the canonical Hermitian connection (cf. [G5, pp. 194-196; K5, pp. 37-39 and W5, pp. 73-86]). Given a $k$-dimensional complex subspace $V$ of $T_{p} M(p \in M)$, we introduce a reduced Ricci form $\operatorname{Ric}_{V}$ on $V$ as follows. Let $e_{1}, \ldots, e_{k}$ be any orthonormal basis of $V$; we may assume that they are part of an orthonormal basis $e_{1}, \ldots, e_{n}$ of $T_{p} M$. Let $\Omega_{i j}$ be the curvature form relative to the frame $\left\{e_{i}\right\}$. Then for $X \equiv \sum_{\alpha=1}^{k} X^{\alpha} e_{\alpha}$ and $Y \equiv \sum_{\alpha=1}^{k} Y^{\alpha} e_{\alpha}$ in $V$, we define

$$
\operatorname{Ric}_{V}(X, Y) \equiv \sum_{\alpha, \beta=1}^{k} X^{\alpha} \bar{Y}^{\beta}\left(\sum_{\gamma=1}^{k} \Omega_{\alpha \beta}\left(e_{\gamma}, e_{\gamma}\right)\right) .
$$

Straightforward algebraic manipulation shows that this definition is independent of the choice of $\left\{e_{1}, \ldots, e_{k}\right\}$. Recall that the bisectional curvature of $X$ and $Y$ is just $K(X, Y) \equiv \sum_{\alpha, \beta=1}^{k} X^{\alpha} \bar{X}^{\beta} \Omega_{\alpha \beta}(Y, Y)$. Hence,

$$
\operatorname{Ric}_{V}(X, X)=\sum_{\alpha=1}^{k} K\left(X, e_{\alpha}\right)
$$

for any orthonormal basis $\left\{e_{1}, \ldots, e_{k}\right\}$ of $V$. Also recall the basic Bochner inequality (first proved in [B2] and subsequently rediscovered in [G5 and K4], among others): if $N$ is a local complex submanifold of $M, p \in N, X, Y \in T_{p} N$ and $K_{N}(X, Y)$ denotes the bisectional curvature of $X$ and $Y$ in the induced metric of $N$, then

$$
K_{N}(X, Y) \leqslant K(X, Y) .
$$

We can now introduce the basic concept underlying this section.

DEFINITION 4.4. $M$ is said to possess strongly negative kth Ricci curvature iff there exists a constant $\Gamma>0$ such that for any $p \in M$ and for any $k$-dimensional complex subspace $V$ of $T_{p} M$, (i) $\operatorname{Ric}_{V}$ is negative definite on $V$, and (ii) $\left|\operatorname{det} \operatorname{Ric}_{V}\right| \geqslant \Gamma$.

The determinant det $\mathrm{Ric}_{V}$ above is of course taken with respect to the given Hermitian metric, i.e. if $\left\{e_{1}, \ldots, e_{k}\right\}$ is any orthonormal basis of $V$, then by definition,

$$
\operatorname{det} \operatorname{Ric}_{V}=\operatorname{det}\left\{\operatorname{Ric}_{V}\left(e_{\alpha}, e_{\beta}\right)\right\}_{1 \leqslant \alpha, \beta \leqslant k}
$$

The purpose of this section is to prove

THEOREM 4.5. Let $M$ be a Hermitian manifold with strongly negative kth Ricci curvature. Then $M$ is strongly k-measure hyperbolic.

The proof of this theorem, which is no more than a simple exercise, will be given at the end of the section. More interesting are the implications of this simple result 
as well as its relationship with the curvature conditions studied by the other authors. These we now explore.

Suppose $k=1$. Then strongly negative 1st Ricci curvature is just the condition that the holomorphic sectional curvature is bounded above by a negative constant. Moreover, strongly 1-measure hyperbolic is, according to Royden [R2], equivalent to hyperbolic in the sense of Kobayashi [K5]. Thus Theorem 4.5 contains the classical result of Grauert-Reckziegel [G2] that holomorphic sectional curvature $\leqslant-\Gamma<0$ implies hyperbolicity. (Note that for a noncompact manifold, there is a strictly stronger result; see Theorem $\mathrm{E}$ in [G4].)

Next suppose $k=n=\operatorname{dim} M$. Then strongly negative $n$th Ricci curvature is equivalent to the following: if $\left\{\lambda_{i}\right\}$ are the eigenvalues of the Ricci tensor, then $\lambda_{i}<0$ for each $i$ and $\left|\lambda_{1} \cdots \lambda_{n}\right| \geqslant \Gamma>0$. In this case, Theorem 4.5 says that if the Ricci tensor of $M$ satisfies these conditions, then $M$ is strongly measure hyperbolic. Two comments are very pertinent at this point. The first is that the preceding statement implies Kobayashi's theorem [K5, p. 121] to the effect that if the Ricci curvature of $M$ is bounded above by a negative constant, then $M$ is strongly measure hyperbolic. A second comment is that this special case of Theorem 4.5 (for $k=n$ ) was in fact stated without proof on p. 373 of [K6]; without being aware of this, Greene and $\mathrm{Wu}$ proved this special case in the process of proving something else [G4, p. 134] and noted explicitly that this criterion of strong measure hyperbolicity "has never been stated in the literature to the best of our knowledge". This is the right place to correct this oversight.

In [C2], J. Carlson proved that if $\Lambda^{k} T M$ is negative, then $M$ is strongly $k$-measure hyperbolic. In our present terminology, the negativity of $\Lambda^{k} T M$ means precisely this: there exists a negative constant $-c$ such that for any $p \in M$, for any $k$-dimensional complex subspace $V$ of $T_{p} M$ and for any unit vector $X \in T_{p} M$, $\sum_{\alpha=1}^{k} K\left(X, e_{\alpha}\right) \leqslant-c$, where $\left\{e_{\alpha}\right\}$ is any orthonormal basis of $V$. In this case, (4.2) implies that $M$ possesses strongly negative $k$ th Ricci curvature. Thus Theorem 4.5 implies Carlson's result. It should also be pointed out that with the stronger condition of the negativity of $\Lambda^{k} T M$, Carlson could prove an extension theorem [C2, p. 279]; with the weaker condition of strongly negative $k$ th Ricci curvature we do not yet see how to recover Carlson's result.

In [S1], B. Shiffman studied a curvature condition which, in our context, may be phrased as follows: for any $p \in M$ and for any $k$-dimensional complex subspace $V$ of $T_{p} M, \operatorname{Ric}_{V}$ has at least one nonpositive eigenvalue. For $k=1$, this is precisely the statement that $M$ has nonpositive holomorphic sectional curvature. It is clear that for each $k$, Shiffman's condition is strictly weaker than ours, and that his condition cannot be used to prove any kind of $k$-measure hyperbolicity result.

It was pointed out that "Ricci curvature $\leqslant-\Gamma<0$ " implies strongly negative $n$th Ricci curvature. Trivially the converse holds on compact manifolds, but in general one can easily construct examples of noncompact $n$-dimensional manifolds of strongly negative $n$th Ricci curvature whose Ricci curvature is not bounded above by any negative constant.

Finally we give the simple proof of Theorem 4.5. Let us begin with a simple observation, which is an immediate consequence of (4.2) and (4.3). 
LeMma 4.6. Let $M$ be a Hermitian manifold with strongly negative kth Ricci curvature with constant $\Gamma$ as in Definition 4.4. If $N$ is a local complex submanifold of $M$ of dimension $\geqslant k$, then $N$ in the induced metric also possesses strongly negative $k$ th Ricci curvature with constant $\Gamma$.

Proof of Theorem 4.5. By hypothesis, $M$ has strongly negative $k$ th Ricci curvature with constant $\Gamma$, for some $\Gamma>0$. Given $p \in M$ and $\alpha \in D_{p}^{k} M$ such that $\|\alpha\|=1$, we must give a positive lower bound of $E_{k}(p ; \alpha)$ as $p$ varies in a neighborhood. Let $f: B_{k} \rightarrow M$ be a holomorphic map such that $f(0)=p$ and $d f(\gamma)=\alpha$ for some $\gamma \in D_{0}^{k} B_{k}$. Let $G$ be the Hermitian metric on $M$. Then $f^{*} G$ is a Hermitian metric on $B_{k}-S$, where $S$ is the hypersurface on which $d f$ is singular; moreover, $f^{*} G$ is a $C^{\infty}$ Hermitian tensor on $B_{k}$ which degenerates on $S$. Thus $f^{*} G$ is a pseudo-hermitian metric on $B_{k}$ in the terminology of [G4] on p. 129. By Lemma 4.6, the restriction of $f^{*} G$ to $B_{k}-S$ has strongly negative $k$ th Ricci curvature with constant $\Gamma$. By the Ahlfors-type lemma on volume forms, due essentially to Chern and Kobayashi (to be precise, the form of the lemma needed for the application here is proved in [G4, Lemma 7.17]), $\Omega_{f^{*} G} \leqslant \Gamma^{-1} \Omega_{k}$, where $\Omega_{k}$ is the volume form of the Bergman metric on $B_{k}$ normalized so that the Ricci curvature is -1 , and where $\Omega_{f^{*} G}$ is the volume form of $f^{*} G$. Note that $\Omega_{f^{*} G}=\left(f^{*} \omega\right)^{k} / k$ !, where $\omega$ is the associated Kähler form of $G$ on $M$. Now

$$
\Omega_{k}=\frac{2^{k}(k+1)^{k}}{\left(1-\|z\|^{2}\right)^{k+1}} \Theta_{k}
$$

(see (2.3) and (2.10)). Hence,

$$
\begin{aligned}
\|\gamma\|^{2} & =2^{k}\left|\Theta_{k}(\gamma \wedge \bar{\gamma})\right|=(k+1)^{k}\left|\Omega_{k}(\gamma \wedge \bar{\gamma})\right| \\
& \geqslant \frac{1}{k !}(k+1)^{k} \Gamma\left|\omega^{k}(\alpha \wedge \bar{\alpha})\right|=(k+1)^{k} \Gamma
\end{aligned}
$$

which shows that $E_{k}(p ; \alpha) \geqslant(k+1)^{k} \Gamma$. Q.E.D.

REMARK. The concept of strongly negative $k$ th Ricci curvature as introduced above is not well understood. It is not known if the existence of a Hermitian metric of strongly negative $k$ th Ricci curvature on a manifold necessarily implies the existence of one with strongly negative $(k+1)$ th Ricci curvature. In fact it is still unknown if a manifold carrying a metric of holomorphic sectional curvature bounded above by a negative constant must also carry a metric of Ricci curvature bounded above by a negative constant (cf. the discussion in [K6, p. 374]). It has been mentioned above that using a stronger geometric assumption (that $\Lambda^{k} T M$ be negative), Carlson [C2] proved an extension theorem for holomorphic mappings from $k$-dimensional manifolds into $M$. It is important to find a proof valid for all $k$ of a similar extension theorem using only the weaker condition of strongly negative $k$ th Ricci curvature. This would clarify the extension theorem for hyperbolic manifolds of Mrs. Kwack and P. J. Kiernan [K9 and K1] on the one hand and the extension theorem for algebraic manifolds of general type due to GriffithsKobayashi-Ochiai-Kodaira [G6, K7 and K8] on the other. The latter extension is in fact the topic of the next section. 
ADDED IN PROOF. It has come to our attention that the material in $\$ 4$ is closely related to a paper of Noguchi [N]. In particular he has introduced a notion similar to the strong negativity of the $k$ th Ricci curvature as defined in $\$ 4$ (cf. [N, p. 414]). Also Theorem 3.1 of Noguchi is relevant to the extension problem posed in the remark at the end of $\S 4$.

5. Extension of holomorphic mappings. In this section, we consider the extension problem of a holomorphic map $f: X-V \rightarrow M$, where $X$ and $M$ are $n$-dimensional complex manifolds with $M$ compact and measure hyperbolic, and $V$ is a proper subvariety of $X$. Under various additional conditions, we show that $f$ can be extended meromorphically across $V$ (Theorem 5.8). When no additional hypotheses are imposed, the most natural approach is to try to exploit the volume-decreasing property of such an $f$. However, we shall give examples of volume-decreasing holomorphic maps between Kähler manifolds for which no such extension is possible. At the end of the section, we discuss some open problems.

First we give some definitions. Let $f: X \rightarrow M$ be a holomorphic mapping between Hermitian manifolds and let $\operatorname{dim} X=k$ and $\operatorname{dim} M=n$, with $k \leqslant n$. Given $p \in X$, let $\left\{\chi_{1}, \ldots, \chi_{k}\right\}$ and $\left\{\mu_{1}, \ldots, \mu_{n}\right\}$ be orthonormal bases of the dual tangent spaces $T_{p}^{*} X$ and $T_{f(p)}^{*} M$ respectively. Define a matrix $A=\left\{A_{\alpha}^{j}\right\}$ by

$$
f^{*} \mu_{\alpha}=\sum_{i=1}^{k} A_{\alpha}^{i} \chi_{i}
$$

for $\alpha=1, \ldots, n$. Let $G$ be the Hermitian metric of $M$; then $G=\sum_{\alpha=1}^{n} \mu_{\alpha} \bar{\mu}_{\alpha}$ at the point $f(p)$. Hence the Hermitian form $f^{*} G$ on $T_{p} X$ may be written

$$
f^{*} G(p)=\sum_{i, j}\left(\sum_{\alpha} A_{\alpha}^{i} \bar{A}_{\alpha}^{j}\right) \chi_{i} \bar{\chi}_{j} .
$$

Let the eigenvalues of the Hermitian form $f^{*} G(p)$ be arranged in increasing order:

$$
0 \leqslant \lambda_{1} \leqslant \cdots \leqslant \lambda_{k} .
$$

For a later reference, we note explicitly that there is an orthonormal basis $\left\{\theta_{1}, \ldots, \theta_{k}\right\}$ of $T_{p}^{*} X$ such that

$$
f^{*} G(p)=\sum_{i=1}^{k} \lambda_{i} \theta_{i} \bar{\theta}_{i} .
$$

Now the map $f$ is said to be quasiconformal iff in the preceding notation there is a positive constant $K$ such that the inequality $\lambda_{k} \leqslant K^{2} \lambda_{1}$ holds at every $p \in M$. For precision, we shall at times refer to this condition as $K$-quasiconformality. In view of (5.2), $f$ being $K$-quasiconformal is equivalent to the validity of the inequality

$$
\max _{\|z\|=1}\|d f(z)\| \leqslant K \min _{\|z\|=1}\|d f(z)\|
$$

at each $p \in X$. A little linear algebra (cf. Lemma 11.1 in [W1]) shows that $f$ is $K$-quasiconformal for some $K>0$ iff there exists a positive constant $K^{\prime}$ such that for all $p \in X$,

$$
\sum_{i, \alpha}\left|A_{\alpha}^{i}\right|^{2} \leqslant K^{\prime}\left|\operatorname{det} f^{*} G(p)\right|^{1 / k},
$$


where $\operatorname{det} f^{*} G(p)$ is understood in the usual sense: $\operatorname{det} f^{*} G(p) \equiv$ $\operatorname{det}\left\{f^{*} G\left(e_{i}, e_{j}\right)\right\}_{1 \leqslant i, j \leqslant k}$ for any orthonormal basis $\left\{e_{1}, \ldots, e_{k}\right\}$ of $T_{p} X$. This can be better said: Recall that the norm $\|d f(p)\|$ of the linear map $d f(p): T_{p} X \rightarrow T_{f(p)} M$ is defined by $\|d f(p)\|^{2}=\sum_{i, \alpha}\left|A_{\alpha}^{i}\right|^{2}$ in the notation of (5.1). Thus $f$ is $K$-quasiconformal for some $K>0$ iff there is a positive constant $K^{\prime}$ such that the inequality

$$
\|d f\|^{2} \leqslant K^{\prime}\left|\operatorname{det} f^{*} G\right|^{1 / k}
$$

holds on $X$. Finally, we say $d f \in L^{p}(X)$ iff $\int_{X}\|d f\|^{p}<\infty$, where the integration is with respect to the volume form of the Hermitian metric on $X$.

TheOREM 5.8. Let $X$ be a $k$-dimensional Hermitian manifold and let $V$ be a proper subvariety of $X$. Let $M$ be an $n$-dimensional compact Hermitian manifold which is strongly $k$-measure hyperbolic, where $k \leqslant n$. Let $f: X-V \rightarrow M$ be a holomorphic mapping. Then $f$ extends to a meromorphic map from $X$ to $M$ if any one of the following conditions is also satisfied:

(i) $f$ is $K$-quasiconformal for some $K>0$.

(ii) $d f \in L^{2 k-2}(X)$.

(iii) $\operatorname{dim} X=\operatorname{dim} M$ and $V$ is a discrete point set in $X$.

Remark. We shall see that the condition in (ii) is in some sense optimal. The condition of $K$-quasiconformality in (i) is very stringent and may even seem somewhat unnatural to some, but it is by now a fairly standard assumption (cf. [W1 and W2], for instance).

Proof. (i) Given $q \in V$ and a neighbourhood $S$ of $q \in X$, it suffices to extend $f$ : $S-V \rightarrow M$ to all of $S$ meromorphically. Using the reasoning in either [G6, pp. 445-446] or [K7, pp. 142-143], we may assume that $S$ is a polydisc $D \times \cdots \times D$ and $S-V$ is a product $\mathbf{D}^{*} \equiv D^{*} \times \cdots \times D^{*} \times D \times \cdots \times D\left(l\right.$ copies of $D^{*} \equiv$ $\{0<|z|<1\}$ and $k-l$ copies of $D$, with $l \leqslant k$ ). To show that $f$ extends to $S$, we again follow [G6] by invoking the extension theorem of Bishop [B1]. Thus, with the understanding that $\mathbf{D}^{*}$ has the Hermitian metric induced from $X$, we are reduced to showing that the graph $\Gamma_{f}$ of $f: \mathbf{D}^{*} \rightarrow M$ has finite volume in the product metric on $D^{*} \times M$. Let $\omega$ and $\Omega$ be the associated Kähler forms of the Hermitian metric on $X$ and $M$ respectively. Then the Kähler form of the induced metric on $\Gamma_{f}$ is (the restriction to $\Gamma_{f}$ of) $\omega+\Omega$, the latter being regarded as defined on $\mathbf{D}^{*} \times M$. Thus:

$$
\text { volume } \Gamma_{f}=\frac{1}{k !} \int_{\mathbf{D}^{*}}\left(\omega+f^{*} \Omega\right)^{k}=\frac{1}{k !} \sum_{j=0}^{k}\left(\begin{array}{l}
k \\
j
\end{array}\right) \int_{\mathbf{D}^{*}} \omega^{k-j} \wedge\left(f^{*} \Omega\right)^{j} .
$$

At a fixed point $p \in \mathbf{D}^{*}$, let $\left\{\theta_{i}\right\}$ be as in (5.4). Then for each $j \geqslant 1$,

$$
\omega^{k-j} \wedge\left(f^{*} \Omega\right)^{j}=(\text { constant })\left(\sum_{i_{1}<\cdots<i_{j}} \lambda_{i_{1}} \cdots \lambda_{i_{j}}\right) \omega^{k} .
$$

Since $\|d f(p)\|^{2}=\lambda_{1}+\cdots+\lambda_{k}$, we see that

$$
\omega^{k-j} \wedge\left(f^{*} \Omega\right)^{j} \leqslant c\|d f\|^{2 j} \omega^{k},
$$


where $c$ is a constant depending only on $k$ and $n$, but independent of $p$. Hence, from (5.9),

$$
\text { volume } \Gamma_{f} \leqslant(\text { const })\left(\int_{\mathbf{D}^{*}} \omega^{k}+\sum_{j=1}^{k} \int_{\mathbf{D}^{*}}\|d f\|^{2 j} \omega^{k}\right)
$$

By (5.7), the $K$-conformality of $f$ implies

$$
\text { volume } \Gamma_{f} \leqslant c^{\prime}\left(\int_{\mathbf{D}^{*}} \omega^{k}+\sum_{j=1}^{k} \int_{\mathbf{D}^{*}}\left|\operatorname{det} f^{*} G\right|^{j / k} \omega^{k}\right),
$$

where the constant $c^{\prime}$ is dependent only on $n, k$ and $K$. Now at each $p \in \mathbf{D}^{*}$ we have, using the notation of (5.4), that

$$
\left|\operatorname{det} f^{*} G\right| \omega^{k}=\left(\lambda_{1} \cdots \lambda_{k}\right) \omega^{k}=\frac{1}{k !}\left(\sum_{i} \lambda_{i} \theta_{i} \wedge \bar{\theta}_{i}\right)^{k}=\frac{1}{k !}\left(f^{*} \Omega\right)^{k} .
$$

Hence noting that $\int_{\mathbf{D}^{*}} \omega^{k}<\infty$, we obtain by Hölder's inequality

$$
\text { volume } \Gamma_{f} \leqslant(\text { const })\left(\text { const }+\int_{\mathbf{D}^{*}}\left(f^{*} \Omega\right)^{k}\right) \text {. }
$$

Pick an $r$ so that $0<r<1$, and let successively $D(r) \equiv\{|z|<r\}, D^{*}(r)=D(r) \cap$ $D^{*}$ and $\mathbf{D}^{*}(r) \equiv D^{*}(r) \times \cdots \times D^{*}(r) \times D(r) \times \cdots \times D(r)$. Now,

$$
\int_{\mathbf{D}^{*}}\left(f^{*} \Omega\right)^{k}=\int_{\mathbf{D}^{*}-\mathbf{D}^{*}(r)}+\int_{\mathbf{D}^{*}(r)},
$$

and the first integral is finite because $f$ is defined on $X-V$. Hence,

$$
\text { volume } \Gamma_{f} \leqslant(\text { const })\left(\text { const }+\int_{\mathbf{D}^{*}(r)}\left(f^{*} \Omega\right)^{k}\right) .
$$

Now $M$ is compact, so the unit sphere bundle of $D^{k} M$ is also compact. Thus for all unit vectors $\gamma \in D^{k} M$, there is the inequality $c_{1}\left|\Omega^{k}(\gamma \wedge \bar{\gamma})\right| \leqslant E_{k}^{M}(\gamma)$, where $c_{1}$ is some positive constant independent of $\gamma$. Hence

$$
\left|\left(f^{*} \Omega\right)^{k}(\alpha \wedge \bar{\alpha})\right| \leqslant \frac{1}{c_{1}}\left(f^{*} E_{k}^{M}\right)(\alpha) \leqslant \frac{1}{c_{1}} E_{k}^{D^{*}}(\alpha)
$$

for all unit $k$-vectors $\alpha$ in $D^{*}$. This implies that $\left(f^{*} \Omega\right)^{k} \leqslant \tau / c_{1}$ by the definition of $\tau$, where $\tau$ is the intrinsic volume form of $D^{*}$. So (5.11) implies:

$$
\text { volume } \Gamma_{f} \leqslant(\text { const })\left(\text { const }+\frac{1}{c_{1}} \int_{\mathbf{D}^{*}(r)} \tau\right)<\infty,
$$

where the last inequality is nothing but a restatement of the fact that $\mathbf{D}^{*}(r)$ has finite volume with respect to the intrinsic measure of $\mathbf{D}^{*}$ (see the discussion at the end of §3). This proves (i). 
(ii) Let $d f \in L^{2 k-2}(X)$. We follow the set-up in the preceding proof of part (i) and again try to show that volume $\Gamma_{f}<\infty$. By (5.9) and (5.10),

$$
\begin{aligned}
\text { volume } \Gamma_{f} & \leqslant \text { (const) }\left(\int_{\mathbf{D}^{*}} \omega^{k}+\sum_{j=1}^{k-1} \int_{\mathbf{D}^{*}} \omega^{k-j} \wedge\left(f^{*} \Omega\right)^{j}+\int_{\mathbf{D}^{*}}\left(f^{*} \Omega\right)^{k}\right) \\
& \leqslant \text { (const) }\left(\int_{\mathbf{D}^{*}} \omega^{k}+\sum_{j=1}^{k-1} \int_{\mathbf{D}^{*}}\|d f\|^{2 j} \omega^{k}+\int_{\mathbf{D}^{*}}\left(f^{*} \Omega\right)^{k}\right) \\
& \leqslant \text { (const) }\left(\int_{\mathbf{D}^{*}} \omega^{k}+\int_{\mathbf{D}^{*}}\|d f\|^{2 k-2} \omega^{k}+\int_{\mathbf{D}^{*}}\left(f^{*} \Omega\right)^{k}\right)
\end{aligned}
$$

where the last inequality is due to Hölder's inequality. Now the first integral of the last sum is clearly finite. The finiteness of the second integral follows from the assumption that $d f \in L^{2 k-2}(X)$. The finiteness of the last integral, i.e. $\int_{\mathbf{D}^{*}}\left(f^{*} \Omega\right)^{k}$, under the assumption that $M$ is compact and strongly $k$-measure hyperbolic has been proved in the proof of part (i). Hence volume $\Gamma_{f}<\infty$.

(iii) Assumption as given in the theorem, we are immediately reduced to showing that if $f: \mathbf{D}-\{0\} \rightarrow M$ is holomorphic, where $\mathbf{D}$ is the polydisc $D \times \cdots \times D$ and $\operatorname{dim} \mathbf{D}=\operatorname{dim} M=n$, then $f$ extends to a meromorphic map defined on $\mathbf{D}$. By a theorem of Stoll (Satz 2 of [S5]), it suffices to show that the cardinality of $f^{-1}(y)$ is finite for a set of points $y \in M$ of positive measure in $M$. Let $N(y)$ be the cardinality of $f^{-1}(y)$. A standard fact (cf. [W3, Lemma 2.12]) states that

$$
\int_{\mathbf{D}-\{0\}} f^{*} \tau_{M}=\int_{y \in M} N(y) \tau_{M}(y),
$$

where $\tau_{M}$ is the intrinsic volume form of $M$. We claim that $\int_{\mathbf{D}-\{0\}} f^{*} \tau_{M}<\infty$. Indeed, the integral is equal to the sum $\int_{\mathbf{D}(r)-\{0\}} f^{*} \tau_{M}+\int_{\mathbf{D}-\mathbf{D}(r)} f^{*} \tau_{M}$, where $\mathbf{D}(r)=\left\{\left|z_{i}\right|<\right.$ $r: i=1, \ldots, n\}$ and $0<r<1$. Since $f$ may be assumed to be defined on $\overline{\mathbf{D}}-\{0\}$, $\int_{\mathbf{D}-\mathbf{D}(r)} f^{*} \tau_{M}<\infty$. To analyze $\int_{\mathbf{D}(r)-\{0\}} f^{*} \tau_{M}$, let $\tau$ denote the intrinsic volume form of $\mathbf{D}-\{0\}$. Then the intrinsic volume-decreasing property of holomorphic maps implies that $f^{*} \tau_{M} \leqslant \tau$. Thus it suffices to show $\int_{\mathbf{D}(r)-\{0\}} \tau<\infty$. That this is so is due to the following elementary reason. Let $\tau_{1}$ be the intrinsic volume form of $\mathbf{D}_{1}^{*} \equiv D^{*}$ $\times D \times \cdots \times D$. Then the imbedding $\mathbf{D}_{1}^{*} \subset \mathbf{D}-\{0\}$ implies $\tau \leqslant \tau_{1}$ on $\mathbf{D}_{1}^{*}$. Hence with $\mathbf{D}_{1}^{*}(r) \equiv \mathbf{D}(r) \cap \mathbf{D}_{1}^{*}$, we have

$$
\int_{\mathbf{D}(r)-\{0\}} \tau=\int_{\mathbf{D}_{1}^{*}(r)} \tau \leqslant \int_{\mathbf{D}_{1}^{*}(r)} \tau_{1}<\infty
$$

where the last inequality has already been used in the proof of part (i). This proves the claim.

Thus by (5.12), $\int_{y \in M} N(y) \tau_{M}(y)<\infty$. Now if $N(y) \equiv \infty$ almost everywhere, this inequality would imply $\tau_{M} \equiv 0$ almost everywhere, thereby contradicting the measure hyperbolicity of $M$. Hence $N(y)<\infty$ on a set of positive measure, as desired. Q.E.D.

REMARK. Note that what is really needed for (iii) is only the measure hyperbolicity (rather than the strong measure hyperbolicity) of $M$. 
Corollary 5.13. Let $X$ and $M$ be 2-dimensional Hermitian manifolds, let $M$ be compact and strongly measure hyperbolic, let $V$ be a proper subvariety of $X$ and let $f$ : $X-V \rightarrow M$ be a holomorphic map such that $d f \in L^{2}(X)$. Then $f$ can be extended to a meromorphic map on $X$.

We now comment on the possibility of proving the preceding theorem without any of the supplementary conditions (i)-(iii). Thus consider the following conjecture (cf. [K7]), which has remained open since the papers [G6, K7 and K8] appeared:

Let $X$ and $M$ be $n$-dimensional complex manifolds, let $V$ be a hypersurface in $X$ and let $M$ be compact and measure hyperbolic. Then any holomorphic map $f: X-V \rightarrow M$ extends to a meromorphic mapping $X \rightarrow M$.

Some special cases are known. In case $M$ is Kähler, a remarkable theorem of Siu [S3], which is an extension of earlier results of Griffiths [G7] and Shiffman [S1], implies that if the codimension of $V$ in $X \geqslant 2$, then (5.14) is true regardless of whether $M$ is measure hyperbolic or not. What happens in case $V$ is a hypersurface and $M$ is in addition Kählerian in (5.14) is unknown at present. Even for an algebraic $M$, the truth of (5.14) is conjectural but, at least in this case, entirely plausible. The reason for this state of affairs is a consequence of the three papers [G6, K6 and K7] of Griffiths, Kobayashi-Ochiai and Kodaira; they proved that when $M$ is an algebraic manifold of general type, then $M$ is measure hyperbolic and furthermore, with $f, X$ and $V$ as in (5.14), the mapping $f$ does extend meromorphically to all of $X$. Thus if in (5.14) $M$ is assumed in addition to be algebraic, then (5.14) would be valid provided one can show that a measure hyperbolic algebraic manifold is of general type. The proof of the latter conjecture in dimension 2 was started by M. Green and Griffiths [G3] and completed by D. Mumford and F. Bogomolov independently (we learned of the latter from Robert Friedman).

If $M$ is not assumed to satisfy an additional hypothesis such as being Kählerian or algebraic, there has been thus far no evidence to show that $(5.14)$ would be valid. We are now going to present an example which, while not a counterexample to (5.14), seems to indicate that (5.14) is unlikely to hold in general. From the vast number of similar extension theorems in the theory of hyperbolic manifolds [K1, K2, K6 and K9], one may be allowed to conclude that the truth of (5.14) would be concomitant with the truth of the following stronger assertion: let $X$ and $M$ be $n$-dimensional Kähler manifolds, let $V$ be a proper subvariety in $X$ and let $f: X-V \rightarrow M$ be a holomorphic mapping which decreases the volume forms of $X$ and $M$ (i.e. $f^{*} \Omega_{M} \leqslant$ $c \Omega_{X}$ for some positive constant $c$, where $\Omega_{X}$ and $\Omega_{M}$ are the volume forms of the respective Kähler metrics); then $f$ extends to a meromorphic mapping on $X$. The following example shows that such an assertion cannot possibly be true.

EXAmPle 5.15. Let $D$ be the unit disc and let $D^{*}=D-\{0\}$ as usual. Let $\phi$ : $D^{*} \rightarrow \mathbf{C} / \mathbf{Z}^{2}$ be a holomorphic mapping, where $\mathbf{Z}^{2}$ stands for the integral lattice in $C$; the derivative $\phi^{\prime}$ of $\phi$ (regarded as a holomorphic function indeterminate up to integral multiples) will be required to be everywhere nonzero in $D^{*}$. Let $\mathbf{Z}^{4}$ be the integral lattice in $\mathbf{C}^{2}$ and let $T^{2}$ be the torus $\mathbf{C}^{2} / \mathbf{Z}^{4}$. Now define $f: D^{*} \times D \rightarrow T^{2}$ 
by $f(z, w)=\left(\phi(z), w / \phi^{\prime}(z)\right)$. With the flat metrics on $D^{*} \times D$ and $T^{2}$ understood, a simple computation shows that $f^{*}$ preserves the volume forms. We now specialize $\phi$ to be $\phi(z)=(1 / 2 \pi i) \log z$. Then $f(z, w)=((1 / 2 \pi i) \log z, 2 \pi i z w)$. Let $\Gamma$ be the graph of $f$ in $\left(D^{*} \times D\right) \times T^{2}$. An elementary computation shows that if $0<r<1$ and $A(r) \equiv\{r<|z|<1\}$, then the volume of $\Gamma(r)$, which is defined to be $\Gamma \cap$ $\left[(A(r) \times D) \times T^{2}\right]$, is equal to $\frac{1}{2}|\log r|+($ polynomial in $r)$. Hence volume $\Gamma(r) \rightarrow \infty$ as $r \rightarrow 0$, so that $f$ cannot have any meromorphic extension to $D \times D$.

Note that $\|d f\|^{2}=\left(1 / 4 \pi^{2}\right)\left(1 /|z|^{2}\right)+$ (polynomial in $|z|^{2}$ and $\left.|w|^{2}\right)$. Thus $d f \notin$ $L^{2}\left(D^{*} \times D\right)$ but $d f \in L^{p}\left(D^{*} \times D\right)$ for $0<p<2$. Since $f$ is volume preserving and at the same time not extendible to $D \times D$ meromorphically, Corollary 5.13 is in some sense best possible.

Another example that is similar to the preceding one but throws a different light on the failure of meromorphic extension may be constructed as follows. This time let $\phi$ be a holomorphic function on $D^{*}$ such that $\phi^{\prime}$ is nowhere zero. Let $f: D^{*} \times D \rightarrow \mathbf{C}^{2}$ be defined by $f(z, w)=\left(\phi(z), w / \phi^{\prime}(z)\right)$. Let $i: \mathbf{C}^{2} \rightarrow \mathbf{P}_{2} \mathbf{C}$ be the natural inclusion of $\mathbf{C}^{2}$ into the projective plane. Then $F \equiv i \circ f: D^{*} \times D \rightarrow \mathbf{P}_{2} \mathbf{C}$ is a holomorphic immersion because $d F$ is nowhere singular. Equip $D^{*} \times D$ with the flat metric and $\mathbf{P}_{2} \mathbf{C}$ with the Fubini-Study metric. Then a straightforward computation shows that if $\Psi$ is the volume form of $\mathbf{P}_{2} \mathbf{C}$ while $\Theta \equiv \Theta_{2}$ (cf. (2.3)) is the volume form on $D^{*} \times D$, then

$$
F^{*} \Psi=\frac{1}{\left(1+\|F\|^{2}\right)^{3}} \Theta .
$$

In particular, $F^{*} \Psi \leqslant \Theta$, so that $F$ is volume decreasing. Letting $\phi(z)=\exp (1 / z)$, for instance, we obtain a volume-decreasing holomorphic immersion of $D^{*} \times D \rightarrow$ $\mathbf{P}_{2} \mathbf{C}$ that has no meromorphic extension to $D \times D$.

Of course all preceding considerations are rooted in the extension theorem of $\mathrm{E}$. Bishop [3] on the closure of the graph of a holomorphic map. The belief in the truth of (5.14) is related to the belief, in view of Bishop's theorem, that the graph of a volume-decreasing holomorphic map must behave reasonably. The two preceding examples show that such is not the case, but in each case the map is $\infty$ to 1 . We now show that even the graphs of volume-decreasing holomorphic imbeddings may not behave.

EXAMPLE 5.16. Let $T^{2}$ be the complex torus $\mathbf{C}^{2} / \mathbf{Z}^{4}$, where $\mathbf{Z}^{4}$ is the integral lattice in $\mathrm{C}^{4}$ as usual. We shall produce holomorphic imbeddings $\phi_{n}: D \times D \rightarrow T^{2}$ such that each $\phi_{n}$ is volume decreasing and that as $n \rightarrow \infty$, volume $\left[\phi_{n}(D \times D)\right] \rightarrow 0$ while the volume of the graph of $\phi_{n} \rightarrow \infty$. The definition of $\phi_{n}$ is

$$
\phi_{n}(z, w)=\left(z / n^{2}+2 w / n, n w\right) .
$$

The complex Jacobian determinant of $\phi_{n}$ is $1 / n$ so that, relative to the flat metrics on $D \times D$ and $T^{2}, \phi_{n}$ is volume decreasing. We now show that each $\phi_{n}$ is an imbedding. Suppose $\phi_{n}(z, w)=\phi_{n}\left(z^{\prime}, w^{\prime}\right)$; then

$$
\begin{gathered}
\left(z-z^{\prime}\right) / n^{2}+2\left(w-w^{\prime}\right) / n=0 \quad\left(\bmod \mathbf{Z}^{2}\right), \\
n\left(w-w^{\prime}\right)=0 \quad\left(\bmod \mathbf{Z}^{2}\right) .
\end{gathered}
$$


Since $\left|z-z^{\prime}\right|<2,\left|w-w^{\prime}\right|<2$ and since we may assume $n \geqslant 10$, the first equation implies $\left(z-z^{\prime}\right) / n^{2}+2\left(w-w^{\prime}\right) / n=0$. The second implies $n\left(w-w^{\prime}\right)=\alpha+i \beta$, for some $\alpha, \beta \in \mathbf{Z}$. Hence $\left(z-z^{\prime}\right) / n^{2}=-2(\alpha+i \beta) / n^{2}$ which implies $z-z^{\prime}=$ $-2(\alpha+i \beta)$ which implies $z-z^{\prime}=0$ because if one of $\alpha, \beta$ is nonzero we would have $2>\left|z-z^{\prime}\right|=2|\alpha+i \beta| \geqslant 2$. Hence also $w=w^{\prime}$, which shows $\phi_{n}$ is an imbedding.

An elementary computation shows that the volume of the graph $\Gamma_{n}$ of $\phi_{n}$ is $\pi^{2}\left(n^{2}+1+5 / n^{2}+1 / n^{4}\right)$ while the volume of $\phi_{n}(D \times D)$ is $\pi^{2} / n^{2}$. Thus as $n \rightarrow \infty$, volume $\Gamma_{n} \rightarrow \infty$ while volume $\phi_{n}(D \times D) \rightarrow 0$.

It remains to raise some questions concerning the extension problem. With part (ii) of Theorem 5.8 in mind, we ask for a weaker version of (5.14):

(5.17) Does Corollary 5.13 hold when both $X$ and $M$ are of dimension $n$ ?

The next question is inspired by the Remmert-Stein extension theorem [R1]:

If $f: B_{n}-A \rightarrow M$ is a holomorphic map, where $A$ is a hypersurface in the unit ball $B_{n}$ and $M$ is an $n$-dimensional compact strongly measure hyperbolic manifold, and if $f$ extends meromorphically across a point of each branch of $A$, does $f$ extend meromorphically across $A$ ?

Recall that in $\$ 2$ we have defined a complex manifold $M$ to be almost hyperbolic if there exists a proper subvariety $S \subset M$ on which the infinitesimal RoydenKobayashi metric $d s_{M}^{2}$ vanishes identically and if $M$ is hyperbolic at each point of $M-S$. Such a manifold is of course measure hyperbolic. Hence the following is another watered-down version of (5.14):

Does (5.14) hold if $M$ is compact and almost hyperbolic and $\left.d s_{M-S}^{2}\right|_{M-c}$ decays to zero on $S$ in a suitable manner?

Needless to say, part of the problem is to specify what should qualify as "suitable manner".

6. Regularity properties of the intrinsic volume form. It is the purpose of this section to exhibit some pathological phenomena of the intrinsic volume forms of two domains in $\mathbf{C}^{2}$. As a consequence, we shall see that the intrinsic volume form of a domain of holomorphy may vanish on a nonempty open set without vanishing identically, thereby settling Problems B.1 and B.6 on pp. 401-402 of [K6].

EXAMPLE 6.1. Let $\Omega \equiv\left\{(z, w) \in \mathbf{C}^{2}:|z w|<1\right\}$. $\Omega$ is a domain of holomorphy. Let $\tau$ be the intrinsic volume form of $\Omega$. We shall show that $\tau \equiv 0$ on $\Omega-\{0\}$, but that $\tau(0)>0$. Indeed, let the intrinsic volume form of $\Omega-\{0\}$ be $\tau^{\prime}$. Then $\tau^{\prime}$ vanishes identically because $\mathbf{C}$ acts effectively on $\Omega-\{0\}$ without fixed points by the action: $t \cdot(z, w)=\left(e^{t} z, e^{-t} w\right)$ for all $t \in \mathbf{C}$. (This is the observation of Kobayashi and Bun Wong on p. 371 of [K6]; note that it is important to explicitly exclude the presence of fixed points of the action for this result to be valid. Otherwise, the present example would furnish a counterexample: $\mathbf{C}$ acts on $\Omega$ itself by the same action, 0 is the unique fixed point and $\tau(0)>0$.) Since $\Omega-\{0\} \subset \Omega, \tau \leqslant \tau^{\prime}$ on $\Omega-\{0\}$. Hence $\tau \equiv 0$ on $\Omega-\{0\}$. 
It remains to prove $\tau(0)>0$. Let $\phi: B_{2} \rightarrow \Omega$ be a holomorphic map such that $\phi(0,0)=(0,0)$. By $(2.4 .1)$, it suffices to show that $\|d \phi[\partial / \partial \zeta \wedge \partial / \partial \eta(0,0)]\|$ has an upper bound independent of $\phi\left(\zeta\right.$ and $\eta$ are the coordinates on $\left.B_{2}\right)$. After a rotation of $B_{2}$, we may let

$$
d \phi\left(\frac{\partial}{\partial \zeta}(0,0)\right)=a \frac{\partial}{\partial z}(0,0), \quad d \phi\left(\frac{\partial}{\partial \eta}\right)(0,0)=b \frac{\partial}{\partial z}(0,0)+c \frac{\partial}{\partial w}(0,0)
$$

for some $a, b, c \in \mathbf{C}$. We have to bound $|a c|$ from above. Let $\phi=\left(\phi_{1}, \phi_{2}\right)$. The polydisc $\Delta_{2}(1 / \sqrt{2})$ of radius $1 / \sqrt{2}$ is contained in $B_{2}$. In $\Delta_{2}(1 / \sqrt{2}),(6.2)$ implies that

$$
\phi_{1}(\zeta, \eta)=a \zeta+b \eta+O(2), \quad \phi_{2}(\zeta, \eta)=c \eta+O(2)
$$

where $O(2)$ denotes terms of order $\geqslant 2$ in $\zeta$ and $\eta$. For $t \in D(1 / \sqrt{2}$ ) (the disc of radius $1 / \sqrt{2}$ in $\mathbf{C})$, define $h(t) \equiv \phi_{1}(t, t) \phi_{2}(t, t)$. Then $h(t)=c(a+b) t^{2}+O(3)$. The definition of $\Omega$ above implies that $\left|\phi_{1} \phi_{2}\right|<1$, and hence $|h(t)|<1$. The Cauchy estimates applied to $h(t)$ then lead to

$$
|c(a+b)| \leqslant 2 .
$$

Similarly, we can define a holomorphic function $f: D(1 / \sqrt{2}) \rightarrow \mathbf{C}$ by $f(t)=$ $\phi_{1}(t,-t) \phi_{2}(t,-t)$. Then $f(t)=c(a-b) t^{2}+O(3)$ and consequently $|c(a-b)| \leqslant 2$. Together with (6.3), we obtain:

$$
|a c| \leqslant\left|\frac{1}{2}(a+b) c\right|+\left|\frac{1}{2}(a-b) c\right| \leqslant 2,
$$

as desired. Q.E.D.

REMARK. If $E_{n}$ denotes the $n$th Eisenman intrinsic norm of the preceding domain $\Omega$, then we have shown that $E_{2} \equiv 0$ on $\Omega-\{0\}$ and $E_{2}(0)>0$. Thus we have an example, with $n=2$, of a domain $\Omega$ such that $\log E_{n}$ is not plurisubharmonic on $\Lambda^{n} T \Omega$, thereby answering negatively Problem B.1 on p. 401 of [K6].

EXAMPLE 6.4. Let $\Delta_{2}$ be the unit polydisc in $\mathrm{C}^{2}$ and let $S$ be the infinite strip $\left\{(z, w) \in \mathbf{C}^{2}:|z|<1 / 2\right\}$. Consider the "infinite cross" region $\Omega \equiv \Delta_{2} \cup S$. The intrinsic volume form $\tau$ of $\Omega$ vanishes identically on $S$, for trivial reasons. We are going to show that $E_{1}(p, X) \geqslant c\|X\|^{2}$ for some positive constant $c$ on compact subsets of $\Omega-\bar{S}$, where $E_{1}$ is the first Eisenman intrinsic norm as defined in (2.5). Thus $\Omega$ is hyperbolic at each point of $\Omega-\bar{S}$ and in particular, $\tau>0$ on $\Omega-\bar{S}$. (We do not know what happens on the real hypersurface $\Omega \cap\{|z|=1 / 2\}$.)

Let $\left(z_{0}, w_{0}\right) \in \Omega-\bar{S}$. Then by definition

$$
\left|z_{0}\right|>1 / 2, \quad\left|w_{0}\right|<1 .
$$

Now write the unit sphere of the tangent space at $\left(z_{0}, w_{0}\right)$ as the union of the following two sets:

$$
\begin{aligned}
& A_{1} \equiv\left\{\left(X_{1}, X_{2}\right):\left|X_{1}\right|^{2}+\left|X_{2}\right|^{2}=1,\left|X_{1}\right| \geqslant \varepsilon\right\}, \\
& A_{2} \equiv\left\{\left(X_{1}, X_{2}\right):\left|X_{1}\right|^{2}+\left|X_{2}\right|^{2}=1,\left|X_{2}\right| \geqslant \varepsilon\right\},
\end{aligned}
$$

where $\varepsilon$ is any preassigned small positive constant. Let $X \in A_{1}$. The holomorphic map $\pi: \Omega \rightarrow D(D=$ unit disc $)$ defined by $\pi(z, w)=z$ maps $X \equiv\left(X_{1}, X_{2}\right)$ onto the 
vector $d \pi(X)$ which has length $\left|X_{1}\right| \geqslant \varepsilon$ and which is tangent to $D$ at $z_{0}$. Hence by the area decreasing property of $E_{1}$,

$$
E_{1}^{\Omega}\left(\left(z_{0}, w_{0}\right) ; X\right) \geqslant E_{1}^{D}\left(z_{0} ; \varepsilon U\right) \text { for all } X \in A_{1} \text {, where } U \text { is a }
$$
unit vector with respect to the flat metric on $D$.

Next let $X \in A_{2}$. Let $\phi: D \rightarrow \Omega$ such that $\phi(0)=\left(z_{0}, w_{0}\right)$ and $d \phi(V)=X$. Write $\phi=\left(\phi_{1}, \phi_{2}\right)$. By the definition of $\Omega,\left|\phi_{1}\right|<1$. If also $\left|\phi_{2}\right|<1$, then using $d \phi_{2}(V)=$ $\left(0, X_{2}\right)$ and the Schwarz lemma, we obtain $|V| \geqslant \varepsilon\left(1-\left|w_{0}\right|^{2}\right)^{-1}$. Thus we may assume that for some $p \in D,\left|\phi_{2}(p)\right|=1$. We may also assume that no other $q \in D$ such that $|q|<|p|$ has the property that $\left|\phi_{2}(q)\right|=1$. If we let $D(|p|) \equiv\{z:|z|<|p|\}$, then $\phi(D(|p|)) \subset \Delta_{2}$ and $\phi(p) \in \partial \Delta_{2} \cap S$. In particular,

$$
\left|\phi_{1}(p)\right|<1 / 2 \text {, }
$$

and $\phi_{2}$ maps $D(|p|)$ into $D$. Since $\phi_{2}(0)=w_{0}$ and the derivative satisfies $\left|\phi_{2}^{\prime}(0)\right|=$ $\left|X_{2}\right| /|V|$, the lemma of Schwarz-Pick applied to $\phi_{2}$ at 0 yields

$$
\left|p X_{2}\right| /\left(1-\left|w_{0}\right|^{2}\right) \leqslant|V| \text {. }
$$

Now consider $\phi_{1}: D \rightarrow D$. We have

$$
\phi_{1}(p)-\phi_{1}(0)=\int_{0}^{1} \frac{d}{d t} \phi_{1}(t p) d t=p \int_{0}^{1} \phi_{1}^{\prime}(t p) d t,
$$

so that by (6.7),

$$
\left|z_{0}\right|-1 / 2 \leqslant\left|z_{0}\right|-\left|\phi_{1}(p)\right| \leqslant|p| \cdot \int_{0}^{1}\left|\phi_{1}^{\prime}(t p)\right| d t .
$$

Hence there exists a $q$ on the line segment joining 0 to $p \in D$ such that

$$
\left|z_{0}\right|-1 / 2 \leqslant|p| \cdot\left|\phi_{1}^{\prime}(q)\right| \text {. }
$$

If we apply the Schwarz-Pick lemma to $\phi_{1}$ at $q$, we get

$$
\left|\phi_{1}^{\prime}(q)\right| \leqslant \frac{1-\left|\phi_{1}(q)\right|^{2}}{1-|q|^{2}} \leqslant \frac{1}{1-|p|^{2}} .
$$

By (6.9), $\left|z_{0}\right|-1 / 2 \leqslant|p| /\left(1-|p|^{2}\right)$ which implies

$$
|p| \geqslant \frac{-1+\left[1+4\left(\left|z_{0}\right|-1 / 2\right)^{2}\right]^{1 / 2}}{2\left(\left|z_{0}\right|-1 / 2\right)}=\frac{2\left(\left|z_{0}\right|-1 / 2\right)}{1+\left[1+4\left(\left|z_{0}\right|-1 / 2\right)^{2}\right]^{1 / 2}} .
$$

Since $(1+x)^{1 / 2} \leqslant 1+\frac{1}{2} x$ for $x \geqslant-1$, the above implies $|p| \geqslant\left(\left|z_{0}\right|-\frac{1}{2}\right) /\left(\left|z_{0}\right|+\frac{1}{2}\right)$. Thus making use of (6.8), we finally obtain

$$
|V| \geqslant \frac{\left|X_{2}\right|}{1-\left|w_{0}\right|^{2}} \cdot \frac{\left|z_{0}\right|-1 / 2}{\left|z_{0}\right|+1 / 2}
$$

Since $X \in A_{2},\left|X_{2}\right| \geqslant \varepsilon$. Hence altogether, we have

$$
\begin{aligned}
E_{1}^{\Omega}\left(\left(z_{0}, w_{0}\right) ; X\right) & \geqslant \min \left\{\left(\frac{\varepsilon}{1-\left|w_{0}\right|^{2}}\right)^{2},\left(\frac{\varepsilon}{1-\left|w_{0}\right|^{2}} \cdot \frac{\left|z_{0}\right|-1 / 2}{\left|z_{0}\right|+1 / 2}\right)^{2}\right\} \\
& =\left(\frac{\varepsilon}{1-\left|w_{0}\right|^{2}} \cdot \frac{\left|z_{0}\right|-1 / 2}{\left|z_{0}\right|+1 / 2}\right)^{2}
\end{aligned}
$$


for all $X \in A_{2}$. Recalling (6.5), we see that the right side of (6.10) is a positive constant independent of $\phi$.

It is now clear that if $\left(z_{0}, w_{0}\right)$ varies within a compact subset of $\Omega-\bar{S}$, the right-hand side of each of (6.6) and (6.10) would remain bounded below by a positive constant. This shows that $\Omega$ is hyperbolic at every point of $\Omega-\bar{S}$. Q.E.D.

We should perhaps mention that many examples similar to Example 6.4 can be easily constructed. Here is one: the domain $\Omega \subset \mathbf{C}^{2}$ defined by

$$
\Omega=\{(z, w):|z|<1\}-\{(z, w):|z| \leqslant 1 / 2,|w| \geqslant 1\} .
$$

Then again, the intrinsic volume form vanishes identically on the open set $\Omega \cap$ $\{(z, w):|z|>1 / 2\}$, while $\Omega$ is hyperbolic at each point of the open set $\Omega \cap\{(z, w)$ : $|z|<1 / 2\}$. We do not know what happens on the real hypersurface $\Omega \cap\{(z, w)$ : $|z|=1 / 2\}$.

It is not known if such irregular behaviour of $\tau$ can occur on compact manifolds.

7. Deformations of measure hyperbolic domains. We shall show by examples that, in a precise sense, the set of measure hyperbolic domains (as well as the set of all strongly measure hyperbolic domains) is neither open nor closed in the space of all domains properly contained in $\mathbf{C}^{n}$. In this whole discussion, let it be noted explicitly that the whole space $\mathbf{C}^{n}$ is excluded from our consideration.

Let the following notation be fixed for the remainder of this section:

$\mathscr{P}=$ the set of all domains properly contained in $\mathbf{C}^{n}$.

$\mathscr{M}=$ the set of all measure hyperbolic domains in $\mathscr{P}$.

$\mathscr{S} \mathscr{M}=$ the set of all strongly measure hyperbolic domains in $\mathscr{P}$.

Thus $\mathscr{S} \mathscr{M} \subset \mathscr{M} \subset \mathscr{P}$. Introduce a generalized distance function $\delta: \mathscr{P} \times \mathscr{P} \rightarrow[0, \infty]$ by

$$
\delta\left(\Omega_{1}, \Omega_{2}\right)=\sup \left\{d\left(z, \partial \Omega_{2}\right): z \in \partial \Omega_{1}\right\}+\sup \left\{d\left(\partial \Omega_{1}, z\right): z \in \partial \Omega_{2}\right\}
$$

where $d$ denotes the euclidean distance of $\mathbf{C}^{n}$ and $\partial$ denotes the boundary operator. We now show that $\delta$ satisfies the triangle inequality

$$
\delta\left(\Omega_{1}, \Omega_{2}\right) \leqslant \delta\left(\Omega_{1}, \Omega_{3}\right)+\delta\left(\Omega_{2}, \Omega_{3}\right) .
$$

The proof of (7.2) is entirely elementary but it does require some finesse. Since it is quite short, we will supply the details. To this end, let us denote the first term on the right side of (7.1) by $\delta_{1}\left(\Omega_{1}, \Omega_{2}\right)$ and the second term by $\delta_{2}\left(\Omega_{1}, \Omega_{2}\right)$. Thus (7.1) now reads

$$
\delta\left(\Omega_{1}, \Omega_{2}\right)=\delta_{1}\left(\Omega_{1}, \Omega_{2}\right)+\delta_{2}\left(\Omega_{1}, \Omega_{2}\right) .
$$

To return to (7.2), suppose $\delta\left(\Omega_{1}, \Omega_{2}\right)=\infty$. If $\delta\left(\Omega_{1}, \Omega_{3}\right)=\infty$, then there is nothing to prove. So suppose $\delta\left(\Omega_{1}, \Omega_{3}\right)=\alpha<\infty$. Given $k$, we shall prove that $\delta\left(\Omega_{2}, \Omega_{3}\right) \geqslant k$. Without any loss of generality, we may assume $\delta_{1}\left(\Omega_{1}, \Omega_{2}\right)=\infty$. Thus there exists $z_{1} \in \Omega_{1}$ such that $d\left(z_{1}, \partial \Omega_{2}\right)>k+\alpha$. Let $z_{3} \in \partial \Omega_{3}$ such that $d\left(z_{1}, z_{3}\right)=d\left(z_{1}, \partial \Omega_{3}\right)$, and let $z_{2} \in \partial \Omega_{2}$ such that $d\left(z_{3}, z_{2}\right)=d\left(z_{3}, \partial \Omega_{2}\right)$. By the triangle inequality for $d$,

$$
d\left(z_{1}, z_{3}\right)+d\left(z_{3}, z_{2}\right) \geqslant d\left(z_{1}, z_{2}\right) \geqslant d\left(z_{1}, \partial \Omega_{2}\right)>k+\alpha .
$$

But

$$
d\left(z_{1}, z_{3}\right)=d\left(z_{1}, \partial \Omega_{3}\right) \leqslant \delta_{1}\left(\Omega_{1}, \Omega_{3}\right) \leqslant \delta\left(\Omega_{1}, \Omega_{3}\right)=\alpha .
$$


Hence, $d\left(z_{3}, z_{2}\right)>k+\alpha-d\left(z_{1}, z_{3}\right) \geqslant k$, which immediately implies $\delta\left(\Omega_{3}, \Omega_{2}\right)>$ $k$. This proves (7.2) if $\delta\left(\Omega_{1}, \Omega_{2}\right)=\infty$.

Now suppose $\delta\left(\Omega_{1}, \Omega_{2}\right)<\infty$. Let $z_{1} \in \partial \Omega_{1}$ so that $d\left(z_{1}, \partial \Omega_{2}\right) \geqslant \delta_{1}\left(\Omega_{1}, \Omega_{2}\right)-\varepsilon$ for a given small positive $\varepsilon$. Let $z_{2} \in \partial \Omega_{2}$ such that $d\left(z_{1}, \partial \Omega_{2}\right)=d\left(z_{1}, z_{2}\right)$. Now choose $z_{3} \in \partial \Omega_{3}$ such that $d\left(z_{1}, \partial \Omega_{3}\right)=d\left(z_{1}, z_{3}\right)$ and then let $z_{2}^{\prime} \in \partial \Omega_{2}$ such that $d\left(z_{3}, \partial \Omega_{2}\right)=d\left(z_{3}, z_{2}^{\prime}\right)$. It follows that

$$
\begin{aligned}
d\left(z_{1}, z_{2}\right) & =d\left(z_{1}, \partial \Omega_{2}\right) \leqslant d\left(z_{1}, z_{2}^{\prime}\right) \leqslant d\left(z_{1}, z_{3}\right)+d\left(z_{3}, z_{2}^{\prime}\right) \\
& =d\left(z_{1}, \partial \Omega_{3}\right)+d\left(z_{3}, \partial \Omega_{2}\right) \leqslant \delta_{1}\left(\Omega_{1}, \Omega_{3}\right)+\delta_{1}\left(\Omega_{3}, \Omega_{2}\right),
\end{aligned}
$$

and hence,

$$
\delta_{1}\left(\Omega_{1}, \Omega_{2}\right)-\varepsilon \leqslant \delta_{1}\left(\Omega_{1}, \Omega_{3}\right)+\delta_{1}\left(\Omega_{3}, \Omega_{2}\right)
$$

Since $\varepsilon$ is arbitrarily small, we obtain

$$
\delta_{1}\left(\Omega_{1}, \Omega_{2}\right) \leqslant \delta_{1}\left(\Omega_{1}, \Omega_{3}\right)+\delta_{1}\left(\Omega_{3}, \Omega_{2}\right) .
$$

Similarly, we obtain

$$
\delta_{1}\left(\Omega_{2}, \Omega_{1}\right) \leqslant \delta_{1}\left(\Omega_{2}, \Omega_{3}\right)+\delta_{1}\left(\Omega_{3}, \Omega_{1}\right) .
$$

Since $\delta_{1}\left(\Omega, \Omega^{\prime}\right)=\delta_{2}\left(\Omega^{\prime}, \Omega\right)$ for any two domains $\Omega, \Omega^{\prime} \in \mathscr{P}$, the preceding becomes

$$
\delta_{2}\left(\Omega_{1}, \Omega_{2}\right) \leqslant \delta_{2}\left(\Omega_{3}, \Omega_{2}\right)+\delta_{2}\left(\Omega_{1}, \Omega_{3}\right) .
$$

Adding this to (7.3), we obtain (7.2).

It is trivial to see that (i) $\delta\left(\Omega, \Omega^{\prime}\right) \geqslant 0$, with equality iff $\Omega=\Omega^{\prime}$, and (ii) $\delta\left(\Omega, \Omega^{\prime}\right)=$ $\delta\left(\Omega^{\prime}, \Omega\right)$. Thus $\delta$ is a distance function on $\mathscr{P}$ in the usual sense of point set topology, except that $\delta$ may take the value $\infty$. Nevertheless, the usual reasoning shows that the sets of the form $U(\Omega) \equiv\left\{\Omega^{\prime}: \delta\left(\Omega, \Omega^{\prime}\right)<1 / k\right\}\left(k \in \mathbf{Z}^{+}\right)$form the basis of a topology in $\mathscr{P}$, so that by abuse of terminology, we shall continue to refer to $\delta$ as a metric on $\mathscr{P}$ (Strictly speaking, the sets $\left\{\left(\Omega, \Omega^{\prime}\right): \delta\left(\Omega, \Omega^{\prime}\right)<r\right\}$ for $0<r<\infty$ form a uniformity on $\mathscr{P}$ so that $\delta$ turns $\mathscr{P}$ into a uniform space.) This topology on $\mathscr{P}$ will be understood in the following discussion.

Before getting to the examples, we make a trivial but important observation; it is an immediate consequence of Lemma 2.16(i).

LEMMA 7.4. Let $f: X \rightarrow M$ be a nondegenerate holomorphic mapping between the $n$-dimensional complex manifolds $X$ and $M$. If $M$ is measure hyperbolic, so is $X$. If $M$ is strongly measure hyperbolic and $f$ is an immersion, then $X$ is also strongly measure hyperbolic.

(Recall: $f$ being nondegenerate means $d f$ is nonsingular somewhere.)

EXAmple 7.5. $\mathscr{M}$ is not open in $\mathscr{P}$. To see this, let $\Omega \equiv\left\{(z, w) \in \mathbf{C}^{2}:|z w|<1\right.$, $|z|<1\}$. The holomorphic map $\phi: \Omega \rightarrow D \times D$ given by $\phi(z, w)=(z, z w)$ is nondegenerate. Hence $\Omega$ is measure hyperbolic (Lemma 7.4). Now for each positive integer $k$, let $\Omega_{k} \equiv \Omega \cup\left\{(z, w) \in \mathbf{C}^{2}:|z|<1 / k\right\}$. Clearly $\delta\left(\Omega_{k}, \Omega\right)<2 / k \rightarrow 0$ as $k \rightarrow \infty$. Since each $\Omega_{k}$ fails to be measure hyperbolic, we are done.

EXAmple 7.6. $\mathscr{S} \mathscr{M}$ is not open in $\mathscr{P}$. Let $\Omega \equiv\left\{(z, w) \in \mathbf{C}^{2}:|z w|<1,0<|z|<1\right\}$. The map $\phi: \Omega \rightarrow D^{*} \times D\left(D^{*} \equiv D-\{0\}\right)$ given by $\phi(z, w)=(z, z w)$ is biholomorphic so that $\Omega$ is strongly measure hyperbolic (Lemma 7.4). Let $\Omega_{k}=\Omega \cup$ $\left\{(z, w) \in \mathbf{C}^{2}: 0<|z|<1 / k\right\}$. Then $\Omega_{k}$ is not measure hyperbolic, but as before $\delta\left(\Omega, \Omega_{k}\right) \rightarrow 0$ when $k \rightarrow \infty$. 
EXAMPLE 7.7. $\mathscr{S} \mathscr{M}$ is not closed in $\mathscr{P}$. Let $\Omega \equiv\left\{(z, w) \in \mathbf{C}^{2}: 0<|z w|<1\right\}$. Then $\mathbf{C}$ acts on $\Omega$ without fixed point by the action $t \cdot(z, w)=\left(e^{t} z, e^{-t} w\right)$ for $t \in \mathbf{C}$. Hence the intrinsic volume form of $\Omega$ vanishes identically (see discussion in Example 6.1). Now for every positive integer $k$, let

$$
\Omega_{k} \equiv\left\{(z, w) \in \mathbf{C}^{2}: 0<\left|z^{k} w^{k+1}\right|<1,0<\left|z^{k+1} w^{k}\right|<1\right\} .
$$

The map $\phi: \Omega_{k} \rightarrow D^{*} \times D^{*}$ such that $\phi(z, w)=\left(z^{k} w^{k+1}, z^{k+1} w^{k}\right)$ is a holomorphic immersion. Hence by Lemma $7.4, \Omega_{k}$ is strongly measure hyperbolic. But $\delta\left(\Omega_{k}, \Omega\right) \leqslant$ $2 / k$ by an elementary computation - making use of the following inequalities on $[1, \infty)$ :

$$
\frac{1}{t}-\left(\frac{1}{t}\right)^{(k+1) / k}=\frac{1}{t}\left(1-t^{-1 / k}\right) \leqslant \frac{1}{t} \log t^{1 / k} \leqslant \frac{1}{k} .
$$

Thus $\delta\left(\Omega_{k}, \Omega\right) \rightarrow 0$ as $k \rightarrow \infty$.

ReMARK. Example 7.7 also shows that $\mathscr{M}$ is not closed in $\mathscr{P}$.

Suppose in the definitions of $\mathscr{P}, \mathscr{M}$ and $\mathscr{S} \mathscr{M}$ above we add the requirement that all domains have nonempty $C^{\infty}$ boundary. Then with obvious modifications, the preceding examples can be adapted to suit the new requirement. Suppose now $\mathscr{P}^{\prime}$ is the set of all proper domains of holomorphy in $\mathbf{C}^{n}$, and $\mathscr{M}^{\prime}$ and $\mathscr{S} \mathscr{M}^{\prime}$ are respectively the subsets of measure hyperbolic and strongly measure hyperbolic domains of holomorphy. Then Example 7.7 shows that neither $\mathscr{M}^{\prime}$ nor $\mathscr{S} \mathscr{M}^{\prime}$ is closed in $\mathscr{P}^{\prime}$. We do not know whether $\mathscr{M}^{\prime}$ and $\mathscr{S} \mathscr{M}^{\prime}$ are open in $\mathscr{P}^{\prime}$.

On the other hand, if we define $\mathscr{P}_{1}, \mathscr{M}_{1}$ and $\mathscr{S} \mathscr{M}_{1}$ using compact manifolds, then nothing seems to be known. In particular, is there a characterization of compact measure hyperbolic manifolds analogous to Brody's criterion for compact hyperbolic manifolds [B3]?

8. Domains of finite euclidean volume. It is well known that all bounded domains are both hyperbolic and measure hyperbolic. To look for measure hyperbolic domains which are not hyperbolic, one must go to unbounded domains. In this context, the most natural candidates are therefore the unbounded domains of finite (euclidean) volume. The idea that finite (euclidean) volume should be related to measure hyperbolicity is further reinforced by the following theorem. First recall that if $M$ is a complex manifold and $p \in M$, the indicatrix of $E_{1}$ (the first Eisenman intrinsic norm) at $p$ is the set

$$
I(p) \equiv\left\{X \in T_{p} M: E_{1}(p ; X)<1\right\} .
$$

This is of course the same as the indicatrix of the infinitesimal Kobayashi-Royden metric.

Proposition 8.1. Let $M$ be a Hermitian manifold. (i) If the indicatrix $I(p)$ at $p \in M$ is of finite volume relative to the Hermitian inner product on $T_{p} M$, then $\tau_{M}(p)>0$, where $\tau_{M}$ is the intrinsic volume form of $M$. (ii) If the volume of the indicatrix $I(p)$ is bounded above whenever p varies in a compact subset of $M$, then $M$ is strongly measure hyperbolic.

REMARK. The converse of part (i) is false. To see this, recall that the intrinsic volume form of the domain $\Omega \equiv\{(z, w):|z w|<1\}$ of Example 6.1 is positive at the 
origin. However, the indicatrix $I(0)$ at $0 \in \Omega$ is just $\Omega$ itself and hence has infinite volume (the flat metric on $\Omega$ is of course understood). The fact that $I(0)=\Omega$ is a particular instance of a folklore result for which both K. Diederich and N. Sibony have shown us proofs. It states: Let $\Omega$ be a disked domain of holomorphy in the sense that $z \in \Omega \Rightarrow \alpha z \in \Omega$ for all $\alpha \in \mathbf{C}$ such that $|\alpha| \leqslant 1$. Then the indicatrix at the origin equals $\Omega$.

Proof of Proposition 8.1. (i) We begin with an observation: if $f: B_{n} \rightarrow M$ $(n=\operatorname{dim} M)$ is a holomorphic map such that $f(0)=p$, then $d f$ maps the unit ball $B$ in the tangent space to $B_{n}$ at 0 into $I(p)$. Indeed, if $X \in B$, then $E_{1}^{M}(p ; d f(X)) \leqslant$ $\|X\|^{2}<1$, by the definition (2.4.1). Now with $f$ above, $d f: T_{0} B_{n} \rightarrow T_{p} M$ is a linear map between inner product spaces with the property $d f(B) \subset I(p)$. Hence, if $c=$ volume $I(p)$ and $\beta=$ the determinant of $d f$, then

$$
\begin{aligned}
\frac{2 \pi^{n}}{n !} & =\text { volume } B=|\beta|^{-2} \text { volume }[d f(B)]<|\beta|^{-2} c, \\
& \Rightarrow|\beta|^{2}<c n ! /\left(2 \pi^{n}\right) \Rightarrow E_{n}^{M}(p ; \omega)>2 \pi^{n} /(n ! c),
\end{aligned}
$$

for any unit $n$-vector $\omega$ at $p \in M$. Thus $\tau_{M}(p)>0$. Part (ii) follows immediately from the preceding proof. Q.E.D.

It follows from Proposition 8.1(i) and the folklore result quoted in the previous remark that if $\Omega$ is a disked domain of holomorphy in $\mathbf{C}^{n}$ with finite volume, then $\tau_{\Omega}(0)>0$. In general, it seems reasonable to ask: is a domain in $\mathbf{C}^{n}$ of finite (euclidean) volume necessarily strongly measure hyperbolic? Example 8.6 below shows that this is not so. This example also shows that there are holomorphic maps $\phi$ : $B_{n} \rightarrow C^{n}$ with Jacobian determinant identically equal to 1 such that the volume of $\phi\left(B_{n}\right)$ is arbitrarily small. It also shows that for domains of finite volume, there can be no Schottky-Landau theorem in the sense of [G8, p. 55]. The next two questions are still open:

Is a domain of finite volume measure hyperbolic?

If $\phi: \mathbf{C}^{n} \rightarrow \mathbf{C}^{n}$ is a nondegenerate holomorphic mapping $(n \geqslant 2)$, is the volume of the image $\phi\left(\mathbf{C}^{n}\right)$ necessarily infinite?

We wish to append some comments on (8.3), which is of course weaker than (8.2). According to Kodaira [K8], the volume of the complement of $\phi\left(\mathbf{C}^{n}\right)$ in $\mathbf{C}^{n}(n \geqslant 2)$ can be infinite in a strong sense even for a holomorphic imbedding $\phi$. It seems to us that if the complement of $\phi\left(\mathbf{C}^{n}\right)$ can be so large, then it would not be so surprising that $\phi\left(\mathbf{C}^{n}\right)$ itself can in fact be quite small, i.e. the answer to (8.3) may well be negative. On the other hand, if one believes that the answer to (8.3) is in the affirmative, then a natural approach would be to assume $\phi\left(\mathbf{C}^{n}\right)$ has finite volume and try to use the first main theorem in value distribution theory to deduce a contradiction. While traditional value distribution theory only deals with holomorphic maps taking values in a compact manifold (so that in the case of $\phi: \mathbf{C}^{n} \rightarrow \mathbf{C}^{n}$, one would regard $\mathbf{C}^{n}$ as a subset of $\mathbf{P}_{n} \mathbf{C}$ and consider the composite mapping $\phi$ : $\mathbf{C}^{n} \rightarrow \mathbf{P}_{n} \mathbf{C}$ instead), the problem at hand demands that the target space $\mathbf{C}^{n}$ should be equipped with the flat metric and hence must remain noncompact. It turns out that 
for the special case of $\mathbf{C}^{n}$ as the target space, it is possible to set up a noncompact value distribution theory (essentially a modified version of the appendix in [W2]). Our effort so far in making use of the resulting first main theorem to attack (8.3) has remained unsuccessful and this is in fact a contributing factor in our present belief that the answer to (8.3) may be negative. For future reference, we are now writing down the first main theorem in this context; the proof will be omitted.

With $\phi: \mathbf{C}^{n} \rightarrow \mathbf{C}^{n}$ as above, let $\tau \equiv \sum_{i} z_{i} \bar{z}_{i}$ be the exhaustion function on the domain space $\mathbf{C}^{n}$ and let the sublevel sets of $\tau$ be denoted by $B(s) \equiv\left\{z \in \mathbf{C}^{n}\right.$ : $\tau(z)<s\}$. Let

$$
\omega_{0} \equiv \frac{\sqrt{-1}}{2} \sum_{i} d z_{i} \wedge d \bar{z}_{i}, \quad \Theta \equiv \omega_{\eta}^{n} / n !
$$

and for $a \in \mathbf{C}^{n}$, let

$$
\lambda_{a} \equiv\left[\frac{4}{(n-1) !} \tau+\frac{1}{4(n-1) \pi^{n}} \cdot \frac{1}{|z-a|^{2 n-2}}\right] \omega_{0}^{n-1} .
$$

Furthermore, for each regular value $a$ of the mapping $\phi$, let $n(t, a)$ be the cardinality of $\phi^{-1}(a) \cap B(t)$. Finally let $d^{c} \equiv \sqrt{-1}\left(d^{\prime \prime}-d^{\prime}\right)$. Then for each regular value $a$ of $\phi$,

$$
\int_{0}^{r} n(t, a) d t=\int_{0}^{r} d t \int_{B(t)} \phi^{*} \Theta+S(r, a)
$$

where $S(r, a) \equiv 4 \int_{B(r)} \phi^{*} \lambda_{a} \wedge \omega_{0}-\int_{\partial B(r)} d^{c} \tau \wedge \phi^{*} \lambda_{a}$. (8.4) is the integrated form of the first main theorem.

We now give the example of a domain with finite volume which is not strongly measure hyperbolic. First we construct a special mapping.

EXAMPLE 8.5. We exhibit a holomorphic map $B_{2} \rightarrow \mathbf{C}^{2}$ with Jacobian determinant identically equal to 1 and with an image of arbitrarily small volume. Let $Q$ be the square of side-length equal to 2 around the origin in $\mathbf{C}$, i.e., $Q \equiv\{(x+\sqrt{-1} y) \in \mathbf{C}$ : $|x|<1$ and $|y|<1\}$. Fix a positive integer $n$ and define $\phi: Q \times Q \rightarrow \mathbf{C}^{2}$ by

$$
\phi(z, w)=\left(\frac{1}{2 \pi n} \exp (2 \pi n z), w \exp (-2 \pi n z)\right) .
$$

The Jacobian determinant $J \phi$ of $\phi$ is identically 1 . Moreover, $\phi$ is a covering map onto its image with fundamental domain $R \equiv\{(x+\sqrt{-1} y) \in \mathbf{C}:|x|<1,0 \leqslant y<$ $1 / n\} \times Q$. Since volume $R=8 / n$ and $J \phi \equiv 1$, we see that volume $\phi(Q \times Q)=8 / n$, which is small if $n$ is large. Restricting this $\phi$ to $B_{2}$ then gives the desired map.

EXAMPLE 8.6. We exhibit a domain of finite volume in $\mathbf{C}^{2}$ which fails to be strongly measure hyperbolic. Using notation as in Example 8.5, let $\phi_{n}: Q \times Q \rightarrow \mathbf{C}^{2}$ be defined by

$$
\phi_{n}(z, w)=\left(\frac{1}{2 \pi n^{5 / 2}} \exp \left(2 \pi n^{3} z\right), w \exp \left(-2 \pi n^{3} z\right)\right) .
$$

Then $J \phi_{n} \equiv n^{1 / 2}$, and $\phi_{n}$ is a covering map onto its image with fundamental domain $R_{n} \equiv\left\{(x+\sqrt{-1} y) \in \mathbf{C}:|x|<1,0 \leqslant y<1 / n^{3}\right\} \times Q$. Since volume $R_{n}=8 / n^{3}$, we see that volume $\phi_{n}(Q \times Q)=8 / n^{2}$. 
Now define a domain $\Omega \subset \mathbf{C}^{2}$ by

$$
\Omega=\bigcup_{n=1}^{\infty} \phi_{n}(Q \times Q)
$$

Hence volume $\Omega<\sum_{n=1}^{\infty} 8 / n^{2}<\infty$. Moreover, if $\mathbf{C}^{*} \equiv \mathbf{C}-\{0\}$ as usual, then $\mathrm{C}^{*} \times\{0\} \subset \Omega$. We claim: the intrinsic volume form $\tau$ of $\Omega$ vanishes identically on $\mathrm{C}^{*} \times\{0\}$.

To prove the claim, let $(\zeta, 0) \in \Omega$, where $\zeta \neq 0$. We must show $\tau(\zeta, 0)=0$. Note that $\Omega$ is circular in the sense that $(a, b) \in \Omega \Rightarrow\left(e^{i \theta} a, e^{i \theta} b\right) \in \Omega$ for $\theta \in[0,2 \pi]$. Hence it suffices to take $\zeta$ above to be real and positive, i.e., we need only prove $\tau(r, 0)=0$ for any $r \in(0, \infty)$. Take $n_{0}$ to be a sufficiently large integer such that for all $n \geqslant n_{0}$,

$$
\frac{1}{2 \pi n^{3}} \log \left(2 \pi n^{5 / 2} r\right)<\frac{1}{2}
$$

Denote by $t$ the left side of (8.7). Then $0<t<1 / 2$ and the ball of radius $1 / 2$ around $(t, 0)$ is contained in $Q \times Q$. Let $B_{2}(1 / 2)$ be the ball of radius $1 / 2$ in $\mathbf{C}^{2}$ and let $\psi_{n}: B_{2}(1 / 2) \rightarrow \Omega$ be defined by $\psi_{n}(z, w)=\phi_{n}(t+z, w)$. Note that $\psi_{n}(0,0)=$ $(r, 0)$ and

$$
d \psi_{n}\left(\frac{1}{n^{1 / 2}} \frac{\partial}{\partial z} \wedge \frac{\partial}{\partial w}(0,0)\right)=\frac{\partial}{\partial \zeta} \wedge \frac{\partial}{\partial \eta}(r, 0)
$$

where $(\zeta, \eta)$ are the coordinates on $\Omega \subset \mathbf{C}^{2}$. Since (8.8) holds for all $n \geqslant n_{0}$, $E_{2}^{\Omega}((r, 0) ; \partial / \partial \zeta \wedge \partial / \partial \eta)=0$. Q.E.D.

REMARKS. (1) The method used in Example 6.4 can be used to show that in fact $\Omega$ is hyperbolic at each point outside $\mathrm{C}^{*} \times\{0\}$. Hence this is an almost hyperbolic domain which is not strongly measure hyperbolic. Since we shall present another such example in the next section, we omit the details. (2) If a domain $\Omega$ has finite (euclidean) volume, then no complex Lie group can act on it effectively. If fact suppose $\mathbf{C}$ acts on $\Omega \subset \mathbf{C}^{n}$. Then there exists a small $(n-1)$-ball $B \subset \Omega$ transversal to the orbits of $\mathbf{C}$. There is then a nondegenerate holomorphic map $\phi: C \times B \rightarrow \Omega$ such that

$$
\phi\left(\alpha,\left(z_{1}, \ldots, z_{n-1}\right)\right)=\alpha \cdot\left(z_{1}, \ldots, z_{n}\right)
$$

for each $\alpha \in \mathbf{C}$. Note that $\phi$ has degree 1, so that if $\Theta_{n}$ is the usual volume form on $\Omega$ and $J \phi$ denotes the Jacobian determinant of $\phi$, then

$$
\int_{\phi(\mathbf{C} \times B)} \Theta_{n}=\int_{\mathbf{C} \times B} \phi^{*} \Theta_{n}=\int_{\mathbf{C} \times B}|J \phi|^{2}=\int_{B}\left(\int_{\mathbf{C}}|J \phi|^{2}\right)=\infty,
$$

where the last equality is because $|J \phi|^{2}$ is plurisubharmonic and so $\int_{\mathbf{C}}|J \phi|^{2} \geqslant$ $|J \phi(0)|^{2} \cdot($ volume $\mathrm{C})=\infty$. (We may assume $J \phi(0) \neq 0$ or else use a translation to achieve this.) Thus certainly volume $\Omega=\infty$, a contradiction.

9. Further examples. The main purpose of this section is to compute the intrinsic volume forms of several domains, in particular unbounded domains. 
9.1. An example of a domain $\Omega$ whose intrinsic volume form $\tau$ is everywhere positive but $\Omega$ is not strongly measure hyperbolic. Let $\Omega=\left\{(z, w) \in \mathbf{C}^{2}:|z w|<1,|z|<1\right\}$. We begin by showing that the intrinsic volume form $\tau$ is positive everywhere. Let $\Omega^{*} \subset \Omega$ be defined by $\Omega^{*} \equiv \Omega-\{z=0\}$. We now show $\tau>0$ on $\Omega^{*}$. Indeed the map $\psi: \Omega \rightarrow D \times D$ given by $\psi(z, w)=(z, z w)$ has nonsingular Jacobian everywhere in $\Omega^{*}$. Hence if $\tau_{1}$ is the intrinsic volume form of $D \times D$, the fact that $\psi^{*} \tau_{1} \leqslant \tau$ implies $\tau>0$ on $\Omega^{*}$.

We next prove that $\tau>0$ on $\{z=0\}$. Pick any $w_{0} \in \mathbf{C}$. To show $\tau\left(0, w_{0}\right)>0$, let $f: B_{2} \rightarrow \Omega$ be a holomorphic map such that $f(0,0)=\left(0, w_{0}\right)$. We shall prove that if $(\zeta, \eta)$ are coordinates on $B_{2}$, then

$$
\left\|d f\left(\frac{\partial}{\partial \zeta} \wedge \frac{\partial}{\partial \eta}(0,0)\right)\right\| \leqslant\left(2+2\left|w_{0}\right|\right) .
$$

This is equivalent to showing $\left\|\tau\left(0, w_{0}\right)\right\| \geqslant|\sqrt{-1} / 2|^{2}\left(2+2\left|w_{0}\right|\right)^{-2}$. To prove (9.1), we may assume, after applying a rotation to $B_{2}$ if necessary, that

$$
d f\left(\frac{\partial}{\partial \zeta}(0,0)\right)=a \frac{\partial}{\partial z}\left(0, w_{0}\right), \quad d f\left(\frac{\partial}{\partial \eta}(0,0)\right)=b \frac{\partial}{\partial z}\left(0, w_{0}\right)+c \frac{\partial}{\partial w}\left(0, w_{0}\right)
$$

where $a, b, c \in \mathbf{C}$. Hence (9.1) is equivalent to

$$
|a c| \leqslant\left(2+2\left|w_{0}\right|\right) \text {. }
$$

Let $f=\left(f_{1}, f_{2}\right)$. The bidisc $\Delta_{2}(1 / \sqrt{2})$ of radius $1 / \sqrt{2}$ is contained in $B_{2}$. Now (9.2) implies that we have the following power series expansions inside $\Delta_{2}(1 / \sqrt{2})$ :

$$
\begin{aligned}
& f_{1}(\zeta, \eta)=a \zeta+b \eta+A_{1} \zeta^{2}+A_{2} \eta^{2}+A_{3} \zeta \eta+O(3), \\
& f_{2}(\zeta, \eta)=w_{0}+c \eta+O(2),
\end{aligned}
$$

where $O(2)$ and $O(3)$ denote terms of order 2 and 3 respectively. Let $D(1 / \sqrt{2})$ be the disc of radius $1 / \sqrt{2}$ in C. Define $h: D(1 / \sqrt{2}) \rightarrow \mathbf{C}$ by $h(t)=f_{1}(t, t) \cdot f_{2}(t, t)$. Then

$$
h(t)=w_{0}(a+b) t+\left[c(a+b)+w_{0}\left(A_{1}+A_{2}+A_{3}\right)\right] t^{2}+O(3) .
$$

Since $|h|<1$ (because $|z w|<1$ in $\Omega$ ), the Cauchy estimates imply that

$$
\left|c(a+b)+w_{0}\left(A_{1}+A_{2}+A_{3}\right)\right| \leqslant 2,
$$

which in turn implies that

$$
|c(a+b)| \leqslant 2+\left|w_{0}\left(A_{1}+A_{2}+A_{3}\right)\right| .
$$

Now $\left|f_{1}\right|<1$ (because $|z|<1$ in $\Omega$ ); hence the Cauchy estimates applied to $f_{1}(t, t)$ $=(a+b) t+\left(A_{1}+A_{2}+A_{3}\right) t^{2}+\cdots$ imply that $\left|A_{1}+A_{2}+A_{3}\right| \leqslant 2$. Hence from (9.4), we obtain

$$
|c(a+b)| \leqslant 2+2\left|w_{0}\right| \text {. }
$$

Similarly define a holomorphic function $g: D(1 / \sqrt{2}) \rightarrow \mathbf{C}$ by

$$
g(t)=f_{1}(t,-t) f_{2}(t,-t) \text {. }
$$

The same reasoning then yields $|c(a-b)| \leqslant 2+2\left|w_{0}\right|$. Together with (9.5), we obtain

$$
|c a|=\frac{1}{2}|c(a+b)+c(a-b)| \leqslant 2+2\left|w_{0}\right|
$$

which is (9.3). Thus $\tau>0$ on $\Omega$. 
To show that $\Omega$ is not strongly measure hyperbolic, it suffices to show that for $p \in \Omega-\{z=0\}, \tau(p) \rightarrow 0$ as $p \rightarrow\{z=0\}$. To this end, let $\Omega^{*}=\Omega-\{z=0\}$ as before. The map $\phi: \Omega^{*} \rightarrow D^{*} \times D$ given by $\phi(z, w)=(z, z w)$ is biholomorphic. Hence if $\tau_{2}$ and $\tau_{3}$ are respectively the intrinsic volume forms of $\Omega^{*}$ and $D^{*} \times D$, then $\phi^{*} \tau_{3}=\tau_{2}$. By (3.4),

$$
\tau_{3}=\left(\frac{\sqrt{-1}}{2}\right)^{2} \frac{d \zeta \wedge d \bar{\zeta}}{4(|\zeta| \log |\zeta|)^{2}} \wedge \frac{d \eta \wedge d \bar{\eta}}{\left(1-|\eta|^{2}\right)^{2}}
$$

where $(\zeta, \eta)$ are the coordinates in $D^{*} \times D$. Hence

$$
\tau_{2}=\left(\frac{\sqrt{-1}}{2}\right)^{2} \frac{d z \wedge d \bar{z}}{4(\log |z|)^{2}} \wedge \frac{d w \wedge d \bar{w}}{\left(1-|z w|^{2}\right)^{2}}
$$

In particular, if $p \in \Omega^{*}$, then $\tau_{2}(p) \rightarrow 0$ as $p \rightarrow\{z=0\}$.

Now since $\Omega^{*} \subset \Omega, \tau \leqslant \tau_{2}$ on $\Omega^{*}$. Hence if $p \in \Omega^{*}$ and $p \rightarrow\{z=0\}$, then $\tau(p) \rightarrow 0$. Q.E.D.

Note that we can improve on the preceding example by requiring $\Omega$ to have $C^{\infty}$ boundary: simply round off the "corners" of $\partial \Omega$ at $\{(z, w):|z|=1=|w|\}$. Call this domain $\Omega^{\prime}$; note that we may require $\Omega^{\prime} \subset \Omega$. Then a trivial modification of the preceding argument, when applied to $\Omega^{\prime}$, shows that $\Omega^{\prime}$ has the same property.

9.2. An example of a domain $\Omega$ which is almost hyperbolic but whose intrinsic volume form vanishes on a hypersurface. Let $\Omega=\left\{(z, w) \in \mathbf{C}^{2}:|z|<1,\left|z^{2} w\right|<1\right\}$. The map $\phi: \Omega \rightarrow D \times D$ such that $\phi(z, w)=\left(z, z^{2} w\right)$ has nonsingular differential on $\Omega^{*}=\Omega$ $-\{z=0\}$. Hence $E_{1}^{\Omega} \geqslant \phi^{*} E_{1}^{D \times D}>0$ on $\Omega^{*}$, proving that $\Omega$ is hyperbolic at each point of $\Omega^{*}$. We show next that the intrinsic volume form $\tau$ of $\Omega$ vanishes on $\{z=0\}$. Indeed, given $\left(0, w_{0}\right) \in \Omega$, let $m$ be any integer such that $m>\left|w_{0}\right|$ and let $\phi_{m}: B_{2} \rightarrow \Omega$ be defined by

$$
\phi_{m}(\zeta, \eta)=\left(\zeta / 2 m, m^{2} \eta+w_{0}\right) .
$$

We must first show that $\phi_{m}$ is well defined, i.e., that $\phi_{m}\left(B_{2}\right) \subset \Omega$. Clearly $|\zeta / 2 m|<1$. Furthermore,

$$
\left|\left(\frac{\zeta}{2 m}\right)^{2}\left(m^{2} \eta+w_{0}\right)\right| \leqslant \frac{1}{4}\left|\eta+\left(\frac{w_{0}}{m^{2}}\right)\right|<1,
$$

because $m>\left|w_{0}\right|$. Hence $\phi_{m}\left(B_{2}\right) \subset \Omega$. Now $\phi_{m}(0,0)=\left(0, w_{0}\right)$. Moreover,

$$
d \phi_{m}\left(\frac{\partial}{\partial \zeta}(0,0)\right)=\frac{1}{2 m} \frac{\partial}{\partial z}\left(0, w_{0}\right) \text { and } d \phi_{m}\left(\frac{\partial}{\partial \eta}(0,0)\right)=m^{2} \frac{\partial}{\partial \eta}\left(0, w_{0}\right) \text {. }
$$

Hence

$$
d \phi_{m}\left(\frac{2}{m} \frac{\partial}{\partial \zeta} \wedge \frac{\partial}{\partial \eta}(0,0)\right)=\frac{\partial}{\partial z} \wedge \frac{\partial}{\partial w}\left(0, w_{0}\right),
$$

so that $E_{2}^{\Omega}\left(\left(0, w_{0}\right) ; \partial / \partial \zeta \wedge \partial / \partial \eta\right) \leqslant(2 / m)^{2}$. Since $m$ is arbitrary, $\tau$ vanishes at $\left(0, w_{0}\right)$. Q.E.D.

Again, by rounding off the "corners" of $\partial \Omega$ at $\{(z, w):|z|=|w|=1\}$, we arrive at the same phenomenon with the additional property that $\Omega$ has $C^{\infty}$ boundary. 
REMARK. The techniques used in the two preceding examples can be used to establish the following comprehensive proposition; the details are omitted.

Proposition 9.7. For any positive numbers $s$ and $t$, define $\Omega_{s, t} \equiv\left\{(z, w) \in \mathbf{C}^{2}\right.$ : $\left.|z|<1,|z|^{s}|w|^{t}<1\right\}$. Then:

(i) If $s>t$, then $\Omega_{s, t}$ is almost hyperbolic, but its intrinsic volume form vanishes on $\{z=0\}$.

(ii) If $s \leqslant t$, the intrinsic volume form of $\Omega_{s, t}$ is everywhere positive, but $\Omega_{s, t}$ is not strongly measure hyperbolic.

(iii) If $s \rightarrow u$ and $t \rightarrow v$, then $\delta\left(\Omega_{s, t}, \Omega_{u, v}\right) \rightarrow 0$ ( $\left.\operatorname{see}(7.1)\right)$.

In particular, let $s \rightarrow 1^{+}$. Then $\delta\left(\Omega_{s, 1}, \Omega_{1,1}\right) \rightarrow 0$. But observe that the intrinsic volume form of $\Omega_{1,1}$ is everywhere positive whereas the intrinsic volume form of $\Omega_{s, 1}$ vanishes on $\{z=0\}$ for all $s>1$. Thus the intrinsic volume form exhibits the “jumping phenomenon" under deformation. In general, the intrinsic volume form is upper-semicontinuous under deformation of complex structure (cf. [R2]).

9.3 An example of a domain of holomorphy which is measure hyperbolic but whose infinitesimal Royden-Kobayashi metric is degenerate on a set larger than a subvariety. (We remark that the construction of this example is related to the construction of the domains $\Omega_{1}$ and $\Omega_{2}$ in [D], and that in the same paper a corresponding phenomenon for the Kobayashi pseudometric has already been observed. The domains in [D] are not known to be measure hyperbolic, however.)

On the disc $D(1 / 2)$ of radius $1 / 2$ in $\mathbf{C}$ consider the function

$$
\phi(z)=\sum_{j=1}^{\infty} \varepsilon_{j} \log \left|z-z_{j}\right|,
$$

where $\varepsilon_{j}$ is a sequence of positive rational numbers such that $\sum_{j} \varepsilon_{j}<\infty$, and $\left\{z_{j}\right\}$ is a sequence of points in $D(1 / 2)$ such that $0 \in\left\{z_{j}\right\}$ and $z_{j} \rightarrow 0$. Observe that $\phi$ is a negative subharmonic function which is $-\infty$ only at the $z_{j}$ 's and that the function

$$
\psi(z, w) \equiv|w| \exp \phi(z)=|w| \prod_{j=1}^{\infty}\left|z-z_{j}\right|^{\varepsilon_{j}}
$$

is plurisubharmonic (psh) on $D(1 / 2) \times \mathbf{C}$. (For the latter fact, observe that $\log \psi$ is clearly psh.) Let $\Omega \equiv\left\{(z, w) \in \mathbf{C}^{2}:|z|<1 / 2, \psi(z, w)<1\right\}$. In $\Omega$ there is a family of lines $\mathscr{L} \equiv\left\{(z, w) \in \Omega: z=z_{j}\right.$ for some $\left.j\right\}$. We claim that $\Omega$ is hyperbolic at every point of $\Omega-\mathscr{L}$. The proof is similar to the proof used in Example 6.4 and so may be omitted. Since $\Omega-\mathscr{L}$ is open dense in $\Omega, \Omega$ is measure hyperbolic. Moreover, the Royden-Kobayashi infinitesimal metric clearly vanishes on every vector tangent to $\mathscr{L}$. Since $\mathscr{L}$ is not a subvariety of $\Omega\left(z_{j} \rightarrow 0\right.$ and $\left.(0,0) \in \mathscr{L}\right)$, we are done.

9.4. The intrinsic volume form of $B_{n}-\{0\}$. Let $\tau^{\prime}$ be the intrinsic volume form of $B_{n}^{\prime} \equiv B_{n}-\{0\}$ and let $I_{n}^{\prime}$ be the intrinsic measure on $B_{n}^{\prime}$ defined by $\tau^{\prime}$ (see (2.12)). If $U$ is any small neighbourhood of 0 in $B_{n}$, it has already been pointed out in the proof of Theorem 5.8 that $I_{n}^{\prime}(U-\{0\})<\infty$; this may be interpreted as an upper bound on $\tau^{\prime}$. We now observe that a lower bound on $\tau^{\prime}$ has already been obtained by Eisenman [P]. Our interest in this lower bound stems partly from an open problem 
posed by Kobayashi. In Problem B.4 on p. 404 of [K6], Kobayashi asked if the intrinsic volume form $\tau$ of $B_{n}$, when restricted to $B_{n}^{\prime}$, in fact equals $\tau^{\prime}$. It follows immediately from Eisenman's result in $[\mathbf{P}]$ that the answer is no. (Contrast this situation with the result of $[\mathbf{C 1}]$.) Proposition 3.4 of $[\mathbf{P}]$ states that if $f: M \rightarrow N$ is a holomorphic mapping between hyperbolic manifolds of dimension $n$ and if $f$ is intrinsic-measure preserving in the sense that $I_{n}^{M}(W)=I_{n}^{N}(f(W))$ for all Borel sets $W$ in $M$, then $f$ must be biholomorphic. In view of this theorem, if indeed $\left.\tau\right|_{B_{0}^{\prime}}=\tau^{\prime}$, then the natural injection $B_{n}^{\prime} \subset B_{n}$ would be biholomorphic, a contradiction.

However, Eisenman's proof of Proposition 3.4 in [P] gives a quantitative comparison between $\tau^{\prime}$ and $\left.\tau\right|_{B_{n}^{\prime}}$ In fact, inequality (3.8) on p. 11 of [P] implies that the inequality

$$
\tau^{\prime}(z) \geqslant \frac{\text { const }}{\|z\|} \tau(z)
$$

holds for all $z$ near 0 and $z \neq 0$ (there is a misprint there: " $k_{u}(\dot{p})$ " should read “ $k_{u}^{\prime}(p)$ " instead).

We now give a mild generalization of (9.8). In the polydisc $\Delta_{n}$, let $S$ be the following codimension $k$ complete intersection: $\left\{\left(z_{1}, \ldots, z_{n}\right) \in \Delta_{n}: z_{1}=\cdots=z_{k}=\right.$ $0\}$. Let $\Delta^{0}=\Delta_{n}-S$ and let $E_{k}^{0}$ be the intrinsic $k$ th Eisenman norm of $\Delta^{0}$. Then we claim

$$
E_{k}^{0}\left(z ; \frac{\partial}{\partial z_{1}} \wedge \cdots \wedge \frac{\partial}{\partial z_{k}}(z)\right) \geqslant \frac{\text { const }}{\|z\|}
$$

for all $z$ near $S, z \in \Delta^{0}$. Indeed, we can write $\Delta^{0}=\Delta_{k}^{*} \times \Delta_{n-k}$, where $\Delta_{k}^{*}=$ $\left\{\left(z_{1}, \ldots, z_{k}, 0, \ldots, 0\right):\left|z_{1}\right|<1\right.$ for $\left.i=1, \ldots, k\right\}-\{(0, \ldots, 0)\}$ and $\Delta_{n-k}=$ $\left\{\left(0, \ldots, 0, z_{k+1}, \ldots, z_{n}\right):\left|z_{i}\right|<1\right.$ for $\left.i=k+1, \ldots, n\right\}$. The natural projection $\pi$ : $\Delta^{0} \rightarrow \Delta_{k}^{*}$ gives

$$
E_{k}^{0}\left(z ; \frac{\partial}{\partial z_{1}} \wedge \cdots \wedge \frac{\partial}{\partial z_{k}}\right) \geqslant E_{k}^{*}\left(\pi(z) ; \frac{\partial}{\partial z_{1}} \wedge \cdots \wedge \frac{\partial}{\partial z_{k}}\right),
$$

where $E_{k}^{*}$ is the intrinsic $k$ th norm of $\Delta_{k}^{*}$. By (9.8),

$$
E_{k}^{*}\left(\pi(z) ; \frac{\partial}{\partial z_{1}} \wedge \cdots \wedge \frac{\partial}{\partial z_{k}}\right) \geqslant \frac{\text { const }}{\|\pi(z)\|} \geqslant \frac{\text { const }}{\|z\|},
$$

so that (9.9) follows.

It follows from (9.9) that if $M$ is an $n$-dimensional complex manifold and $V$ is a subvariety of codimension $k$, then $\left.E_{k}^{M}\right|_{M-V} \neq E_{k}^{M-V}$. It is possible that an elaboration of the argument of [C1] would lead to the complementary assertion that, with $M, V$ as above, then $\left.E_{l}^{M}\right|_{M-V}=E_{l}^{M-V}$ for $l=1, \ldots, k-1$.

9.5. All strictly pseudoconvex domains are hyperbolic. The purpose of this section is to make use of the following theorem of Sibony in [S2] to produce a large class of almost hyperbolic manifolds.

THEOREM 9.10 [S2]. If $\Omega$ is a domain in $\mathbf{C}^{n}$ on which is defined a negative continuous psh function $\psi$, then $\Omega$ is hyperbolic at each point of every open subset on which $\psi$ is strictly psh. 
Sibony has proved even sharper statements than this in [S2] (see also the discussion after Problem 2 on p. 141 of [W5]), but Theorem 9.10 is all we need here. A simple proof of this theorem due to Diederich is reproduced in [W5, pp. 141-142]. As an immediate corollary, we obtain

Corollary 9.11. Let $\psi$ be a $C^{2}$ psh function on $\mathbf{C}^{n}$ and for each $c \in \mathbf{R}$, let $\Omega_{c} \equiv\left\{z \in \mathbf{C}^{n}: \psi(z)<c\right\}$. Then $\Omega_{c}$ is hyperbolic at every point where $\psi$ is strictly psh.

We can use this corollary to construct a new class of unbounded measure hyperbolic domains distinct from all those we have encountered so far.

EXAMPLE 9.12. Let $\phi(z)$ be a $C^{\infty}$ subharmonic function of the variable $z$. The function $\psi$ on $\mathbf{C}^{n}$ defined by $\psi(z, w)=|w|^{2} e^{\phi(z)}$ is $C^{\infty}$ psh, and is strictly psh outside the set $L \equiv\{(z, w): w=0$ or $\Delta \phi(z)=0\}$; this is an easy computation. Now suppose

the domain of harmonicity of $\phi$ (i.e. the set of $z \in \mathbf{C}$ such that $\Delta \phi(z)=0)$ is a discrete point set.

Then $L$ is a hypersurface in $\mathbf{C}^{2}, L$ being the union of the $z$-axis together with at most a countable collection of complex lines in $\mathbf{C}^{2}$ each parallel to the $w$-axis and passing through a point of the form $(z, 0)$, where $\Delta \phi(z)=0$. It follows that each sublevel set $\Omega_{c} \equiv\{\psi<0\}$ is hyperbolic in $\Omega_{c}-L$ by Corollary 9.11 , and is almost hyperbolic if (9.13) is satisfied because $\Omega_{c} \cap L$ is a subvariety of $\Omega_{c}$.

More generally, assume instead

the domain of harmonicity of $\phi$ is a set of measure zero in $\mathbf{C}$.

Since $\Omega_{c}$ is still hyperbolic at each point of $\Omega_{c}-L, \Omega_{c}$ is measure hyperbolic: for in this case, $\Omega_{c} \cap L$ is a set of measure zero in $\Omega_{c}$ (cf. Proposition 2.14 of [P]). This remark leads to an easy construction of measure hyperbolic manifolds which are hyperbolic at almost all points but are not necessarily almost hyperbolic: take any $C^{\infty}$ nonnegative function $\rho$ on $\mathbf{C}$ such that the zero set of $\rho$ is nondiscrete but is of measure zero (e.g. a curve in C), let $\phi$ satisfy $\Delta \phi=\rho$, and let $\Omega_{c}$ be constructed using this $\phi$. This $\Omega_{\text {c }}$ is the required measure hyperbolic manifold; it may not be almost hyperbolic in general because $\Omega_{\text {c }}$ is a priori only hyperbolic at each point of $\Omega_{c}-L$, and $L \cap \Omega_{c}$ is a real hypersurface in $\Omega_{c}$ (if we take the zero set of $\rho$ to be a curve in C).

The following proposition sharpens Corollary 9.11 in a specific situation.

Proposition 9.15. Let $\Omega$ be a (possibly unbounded) pseudoconvex domain in $\mathbf{C}^{n}$ such that $\partial \Omega$ is $C^{\infty}$ and is strictly pseudoconvex except on a set $S$ of measure zero in $\partial \Omega$. Then $\Omega$ is measure hyperbolic. If $S=\varnothing$, then $\Omega$ is hyperbolic.

Proof. Let $\delta$ be the distance from $\partial \Omega$, i.e., for each $z \in \Omega, \delta(z)=\operatorname{dist}(z, \partial \Omega)$, with euclidean distance understood. By assertion (P9) on p. 72 of [W4], $e^{-\delta}$ is psh on $\Omega$, and is strictly psh on $\Omega-\Sigma$, where $\Sigma$ is the union of all the lines orthogonal to $\partial \Omega$ and passing through a point of $S$ (the latter assertion follows immediately from the proof of (P9) in [W4]). Now $\Sigma$ is a set of measure zero in $\mathbf{C}^{n}$ because $S$ is a set of measure zero in $\partial \Omega$. By Corollary $9.11, \Omega$ is hyperbolic at each point of $\Omega-\Sigma$ and 
hence measure hyperbolic (again compare Proposition 2.14 of [P]). If $S=\varnothing$, then $\Sigma=\varnothing$, and $\Omega$ is hyperbolic. Q.E.D.

The last assertion of Proposition 9.15, that any $C^{\infty}$ strictly pseudoconvex (bounded or unbounded) domain is hyperbolic, is of course an easy consequence of Sibony's theorem (Theorem 9.10 above). It is quite possible that such domains are actually complete hyperbolic (note that a theorem in [S2] already implies that they are taut in the sense of [W1]). More than this, it is an obvious question as to whether the boundary estimates of the first author's work [G1] can be extended to this case.

\section{BIBLIOGRAPHY}

[B1] E. Bishop, Conditions for the analyticity of certain sets, Michigan Math. J. 11 (1964), 289-304.

[B2] S. Bochner, Curvature in Hermitian manifolds, Bull. Amer. Math. Soc. 53 (1947), 179-195.

[B3] R. Brody, Compact manifolds and hyperbolicity, Trans. Amer. Math. Soc. 235 (1978), 213-219.

[B4] R. Brody and M. Green, $A$ family of smooth hyperbolic hypersurfaces in $\mathbf{P}_{3} \mathbf{C}$, Duke Math. J. 44 (1977), 873-874.

[C1] L. A. Campbell, A. Howard and T. Ochiai, Moving holomorphic discs off analytic subsets, Proc. Amer. Math. Soc. 60 (1976), 106-108.

[C2] J. A. Carlson, Some degeneracy theorems for entire functions with values in an algebraic variety, Trans. Amer. Math. Soc. 168 (1972), 273-301.

[D] K. Diederich and N. Sibony, Strange complex structures on Euclidean space, J. Reine Angew. Math. 311-312 (1979), 397-407.

[E] D. A. Eisenman, Intrinsic measures on complex manifolds and holomorphic mappings, Mem. Amer. Math. Soc., No. 96, Amer. Math. Soc., Providence, R.I., 1970.

[G1] I. Graham, Boundary behavior of the Carathéodory and Kobayashi metrics on strongly pseudoconvex domains in $C^{n}$, Trans. Amer. Math. Soc. 207 (1975), 219-240.

[G2] H. Grauert and H. Reckziegel, Hermitesche Metriken und normale Familien holomorpher Abbildungen, Math. Z. 89 (1965), 108-125.

[G3] M. Green and P. A. Griffiths, Two applications of algebraic geometry to entire holomorphic mappings, The Chern Symposium 1979 (W.-Y. Hsiang et al., Eds.), Springer-Verlag, New York and Berlin, 1980, pp. 41-74.

[G4] R. E. Greene and H. Wu, Function theory on manifolds which possess a pole, Lecture Notes in Math., Vol. 699, Springer-Verlag, New York and Berlin, 1979.

[G5] P. A. Griffiths, Hermitian differential geometry, Chern classes, and positive vector bundles, Global Analysis (Papers in Honor of K. Kodaira), Univ. of Tokyo Press, Tokyo, 1969, pp. 185-251.

[G6] Holomorphic mappings into canonical algebraic varieties, Ann. of Math. 93 (1971), 439-458.

[G7] __ Two theorems on extensions of holomorphic mappings, Invent. Math. 14 (1971), 27-62.

[G8] Entire holomorphic mappings in one and several complex variables, Ann. of Math. Studies, no. 85, Princeton Univ. Press, Princeton, N.J., 1976.

[K1] P. J. Kiernan, Extensions of holomorphic maps, Trans. Amer. Math. Soc. 172 (1972), 347-355.

[K2] _ Holomorphic extension theorems, Value Distribution Theory, Part A, Dekker, New York, 1974, pp. 97-107.

[K3] P. J. Kiernan and S. Kobayashi, Holomorphic mappings into projective space with lacunary hyperplanes, Nagoya Math. J. 50 (1973), 199-216.

[K4] S. Kobayashi, Invariant distances on complex manifolds and holomorphic mappings, J. Math. Soc. Japan 19 (1967), 460-480.

[K5] __ Hyperbolic manifolds and holomorphic mappings, Dekker, New York, 1970.

[K6] _ Intrinsic distances, measures and geometric function theory, Bull. Amer. Math. Soc. 82 (1976), 357-416.

[K7] S. Kobayashi and T. Ochiai, Mappings into compact complex manifolds with negative first Chern class, J. Math. Soc. Japan 23 (1971), 137-148.

[K8] K. Kodaira, Holomorphic mappings of polydiscs into compact complex manifolds, J. Differential Geometry 6 (1971-72), 33-46.

[K9] M. H. Kwack, Generalization of the big Picard theorem, Ann. of Math. 90 (1969), 9-22. 
[N] J. Noguchi, Meromorphic mappings into a compact complex space, Hiroshima Math. J. 7 (1977), $441-425$.

[P] D. A. Pelles (= D. A. Eisenman), Holomorphic maps which preserve intrinsic measure, Amer. J. Math. 97 (1975), 1-15.

[R1] R. Remmert and K. Stein, Über die wesentlichen singularitäten analytischer Mengen, Math. Ann. 126 (1953), 263-306.

[R2] H. L. Royden, Remarks on the Kohavashi metric, Several Complex Variables II (Proc. Internat. Conf. Univ. of Maryland, 1970), Lecture Notes in Math., Vol. 185, Springer-Verlag, New York and Berlin, 1971, pp. 125-137.

[R3] _ The extension of regular holomorphic maps, Proc. Amer. Math. Soc. 43 (1974), 306-310.

[S1] B. Shiffman, Holomorphic and meromorphic mappings and curvature, Math. Ann. 222 (1976), 171-194.

[S2] N. Sibony, A class of hyperbolic manifolds, Recent Developments in Several Complex Variables (J. E. Fornaess, Ed.), Ann. of Math. Studies, no. 100, Princeton Univ. Press, Princeton, N.J., 1981.

[S3] Y. T. Siu, Extension of meromorphic maps into Kähler manifolds, Ann. of Math. 102 (1975), $421-462$.

[S4] _ Every Stein subvariety admits a Stein neighbourhood, Invent. Math. 38 (1976), 89-100.

[S5] W. Stoll, Über die Fortsetzharkeit analytischer Mengen endlichen Oberflacheninhaltes, Arch. Math. 9 (1958), 167-175.

[W1] H. Wu, Normal families of holomorphic mappings, Acta Math. 119 (1967), 194-233.

[W2] Remarks on the first main theorem in equidistribution theory. III, J. Differential Geometry 3 (1969), 83-94.

[W3] _. The equidistribution theory of holomorphic curves, Ann. of Math. Studies, no. 64, Princeton Univ. Press, Princeton, N.J., 1970.

[W4] _ An elementary method in the study of nonnegative curvature, Acta Math. 152 (1979), $57-78$

[W5] _ Function theory on noncompact Kähler manifolds, Complex Differential Geometry, DMV Seminar, Bd. 3, Birkhäuser Verlag, Basel, 1983, pp. 69-158.

Department of Mathematics, University of Toronto, Toronto, Canada M5S 1A1

Department of Mathematics, University of California, Berkeley, California 94720 